\title{
Effects of dietary supplementation with prebiotics and Pediococcus acidilactici on gut health, transcriptome, microbiome, and metabolome in Atlantic salmon (Salmo salar L) after seawater transfer
}

Anusha K. S. Dhanasiri ( $\nabla$ anusha.dhanasiri@nmbu.no)

Norwegian University of Life Sciences (NMBU)

Alexander Jaramillo-Torres

Norwegian University of Life Sciences (NMBU)

Elvis M. Chikwati

Norwegian University of Life Sciences (NMBU)

Torunn Forberg

BioMar (Norway)

Åshild Krogdahl

Norwegian University of Life Sciences (NMBU)

Trond M. Kortner

Norwegian University of Life Sciences (NMBU)

\section{Research Article}

Keywords: Functional ingredients, prebiotics, probiotics, FOS, GOS, gut microbiota, Pediococcus acidilactici, metabolomics, transcriptomics, Atlantic salmon

Posted Date: September 7th, 2022

DOI: https://doi.org/10.21203/rs.3.rs-1163708/v2

License: (c) (i) This work is licensed under a Creative Commons Attribution 4.0 International License.

Read Full License 


\section{Abstract \\ Background}

Given the importance of gut microbiota for health, growth and performance of the host, the aquaculture industry has taken measures to develop functional fish feeds aiming at modulating gut microbiota and inducing the anticipated beneficial effects. However, present understanding of the impact of such functional feeds on the fish is limited. The study reported herein was conducted to gain knowledge on performance and gut health characteristics in post-smolt Atlantic salmon fed diets varying in content of functional ingredients. Three experimental diets, a diet containing fructo-oligosaccharides (FOS), a diet with a combination of FOS and Pediococcus acidilactici (BC) and a diet containing galactooligosaccharides (GOS) and BC, were used in a 10-weeks feeding trial. A commercial diet without functional ingredients was also included as a control/reference. Samples of blood plasma, mucosa and digesta were subjected to microbiome, transcriptome and metabolome profiling for evaluation of the diet effects.

\section{Results}

No significant growth differences were observed between fish fed the supplemented diets, but FOS-BC fed fish showed significantly faster growth than the control fed fish. The microbiome results showed that the $B C$ was present in both the digesta, and the mucosa samples of fish fed the FOS-BC and GOS-BC diets. Digesta-associated microbiota was altered, while mucosa-associated microbiota was relatively unaffected by diet. Replacing FOS with GOS increased the level of metabolites linked to phospholipid, fatty acid, carnitine and sphingolipid metabolism. Variation in metabolite levels between the treatments closely correlated with genera mainly belonging to Firmicutes and Actinobacteria phyla. The transcriptome analyses indicated diet effects of exchanging FOS with GOS on immune functions, oxidative defense and stress responses. No significant diet effect was observed on intestinal inflammation in the pyloric ceca or in the distal intestine, and no steatosis was observed in the pyloric ceaca.

\section{Conclusions}

Dietary supplementation with $\mathrm{BC}$ induced moderate effects on the microbiota of the digesta, while the effects of replacing FOS with GOS were more marked and was observed also for nutrient metabolism. Our data indicates therefore that the quality of a prebiotic may be of great importance for the effects of a probiotic on gut microbiota, function, and health.

\section{Background}


To be able to grow sustainably, the salmon aquaculture industry has during the last two decades moved away from the traditional high fishmeal/fish oil diets, by gradually increasing the use of plant raw materials and alternative sources of lipid. Dietary incorporation of functional ingredients is also gaining attention to improve the robustness of the fish. Gut microbiota is important for performance and wellbeing of the fish. Therefore, efforts have been made to develop functional feeds aiming at modulating the gut microbiota to induce anticipated beneficial effects. Several previous studies have been conducted to evaluate the effect of feeds supplemented with probiotics, prebiotics or synbiotics, i.e. combinations of pre and pro-biotics, for farmed fish species including Atlantic salmon [1-5]. However, further efforts are still needed to better understand the combined effect of those functional ingredients on gut microbiota, gut function and health, and overall performance of the fish.

Dietary supplementation of probiotic bacteria can modulate gut microbiota and gut immune responses in beneficial ways and contribute to the synthesis of nutrients, ultimately improving disease resistance and growth performance of the fish [1]. The lactic acid bacteria, Pediococcus acidilactici MA 18/5M, is among the most widely studied probiotic bacteria for farmed fish species [6-10] and has been reported to enhance gut mucosal and peripheral immunity. Prebiotics may also exert beneficial host effects, via stimulation of the growth and/or the activity of the gut microbial population [3]. Several studies have indicated beneficial effects in fish of prebiotics such as fructo-oligosaccharide (FOS), galactooligosaccharide (GOS), mannan-oligosaccharide, beta glucans and inulin $[3,4,11]$. On the other hand, a recent large-scale study with salmon under commercial farming conditions showed little or no effects of dietary supplementation of a mixture of nucleotides, yeast cell walls and essential fatty acids [12], but indicated that these specific functional ingredients may represent an energetic cost for the fish.

Synbiotics, a mixture of probiotic and prebiotic agents, can have beneficial effects on the host by improving the survival and implantation of probiotic and/or the growth and activity of the indigenous beneficial bacteria in the gut [13]. Therefore, an optimal combination of probiotics and prebiotics in a single product could elicit a superior effect, compared to the activity of each component alone [14]. Studies of application of synbiotics in aquaculture species have increased over the past years including some studies on salmonids $[2,5,15,16]$. Dietary application of $P$. acidilactici and GOS has shown effects such as increased immune responses and disease resistance, microbiota and metabolic alterations in rainbow trout (Oncorhynchus mykiss) [17-19], increased growth in juvenile rockfish (Sebastes schlegeli) and some effects on mucosal and serum immune parameters in common carp (Cyprinus carpio) fingerlings [20]. A few studies have reported effects of dietary inclusion with P. acidilacticiand FOS such as modulation of gut microbiota and immunity in Atlantic salmon [7] and increased growth performance of Caspian roach (Rutilus frisii kutum) fry [21].

Economically, Atlantic salmon is one of the most important farmed fish species worldwide [22]. The postsmolt stage (early marine phase) is one of the critical stages in Atlantic salmon life cycle [23]. Suppression of gut health [12] and alterations of gut microbiota [24] were reported in Atlantic salmon during early marine phase. In this stage functional feeds could play an important role in increasing survival, health, growth, and overall performance of the fish. Considering the importance of gut 
microbiota in modulating the gut health and ultimately overall health and performance of the fish, this study was conducted to evaluate effects in post smolt Atlantic salmon of supplementing a diet containing the prebiotic FOS with the probiotic $P$. acidilactici $(\mathrm{BC})$ and replacing FOS in the diet containing $\mathrm{BC}$ with GOS. A grower diet without functional ingredients was also included as a reference/control. An overview of the experimental set up and investigated endpoints is illustrated in Fig. 1 and detailed in the materials and methods section. A multi-omics analytical approach was chosen with microbiome, transcriptome and metabolome profiling. This study strengthens the knowledge basis of effects of use of functional feeds on fish by unveiling the complex interrelated associations among the gut microbiotatranscriptome-metabolites. It will also aid in the generation of novel biomarkers which could enhance the potential application of functional diets into the commercial feed formulations.

\section{Results}

The following outline of the presentation of the results has been chosen; firstly, to give a picture of the overall results from all treatments, before more detailed comparisons are made between the two pairs of treatment for which the cause of differences can be interpreted and discussed to achieve the goals of the study, i.e. fish fed the FOS and the FOS-BC diets and those fed the FOS-BC and GOS-BC diets. This approach will help us to understand the effects of supplementation of probiotic, BC, to prebiotic, FOS, and the influence of alteration of prebiotic combined with BC.

\section{Overall performance}

The fish grew well throughout the experiment showing thermal growth coefficients (TGCs) averaging about 3.1 (Fig. 2). Fish in the FOS-BC group grew significantly faster than those in the control group, showing TGCs of 3.23 and 2.96, respectively, during the 10 weeks of feeding. Fish in the other treatments showed intermediate TGCs. Feed intake and feed conversion ratios, which averaged $847 \mathrm{~g} \pm 8$ (SEM) and $1.12 \pm 0.02$, respectively, showed no significant differences among the four treatments.

\section{Overall results of gut histology}

The distal intestine and pyloric caeca of the fish from the four treatments showed largely normal morphological characteristics, but some individuals from all diet groups showed abnormal morphology that ranged from mild to severe. Figures $3 \mathrm{a}$ and $3 \mathrm{~b}$ illustrate the observations made regarding signs of inflammation in the distal intestine, i.e. regarding cell infiltration and loss of distal intestine enterocyte vacuoles, respectively. The results showed no significant differences between treatments. The same was observed regarding infiltration of inflammatory cells in mucosa and lipid accumulation (steatosis) in pyloric caeca, (i.e. inflammation and steatosis, Figs. 3c and 3d, respectively).

\section{Overall results of microbiota profiling}

The absolute bacterial DNA levels measured by qPCR analysis did not show significant differences between any of the three experimental diets. However, the variation between samples within treatment 
was large (Additional File 1. Figure S1). Generally bacterial DNA levels in digesta was higher compared to the levels in mucosa.

Alpha diversity, i.e. number of different species within a sample, measured as observed species and Shannon indices, are presented in Additional File 1: Figures S2a and S2b for digesta and S2c and S2d for mucosa. In the digesta samples, significant differences between the treatments were observed (observed species: $p=0.05$ and Shannon index: $p=0.05$ ). Fish fed the control diet showed the highest value for observed species, those fed the GOS-BC diet showed the lowest with the difference being significant (observed species: $p=0.04$ ). The mucosa samples did not show significant diet effects (observed species: $p=0.22$ and Shannon index: $p=0.09$ ) among the fish fed different diets.

Beta diversity, i.e. differences in bacterial species between samples taking into account species differences as well as the abundance of the species, was evaluated by PERMANOVA analysis based on Bray-Curtis dissimilarity matrix. For the digesta samples (Fig. 4a) significant differences among treatments were observed $\left(F=1.99, R^{2}=0.176\right.$ and $\left.p=0.04\right)$. On the other hand, the mucosa samples (Figs. $4 b)$ did not show significant differences in beta diversity $\left(F=1.24, R^{2}=0.117\right.$ and $p=0.23$ ) among different treatments.

In the digesta, at the phylum level, Firmicutes and Proteobacteria dominated the bacterial communities, representing more than $80 \%$ of the average relative abundance in all treatments (Additional File 1: Figure S3a). At the genus level, the lactic acid bacteria group, represented mainly by Lactobacillus and Leuconostoc comprised around $50 \%$ of the average relative abundance in all treatments (Fig. $5 \mathrm{a}$ ). In the mucosa, the most abundant phyla were Firmicutes and Proteobacteria followed by Tenericutes. Together they accounted for approximately $80 \%$ of averaged relative abundance in all the treatments (Additional File 1: Figure S3b). The dominant genera in mucosa were Lactobacillus, Aliivibrio and Leuconostoc which comprised around $50 \%$ averaged relative abundance per feeding group (Fig. 5b). The complete list of genera in digesta which showed significant changes in their abundance among treatments are presented in Additional File 2: Tables S1.

The Random Forest model performed well for predicting the results of the four treatments regarding the digesta samples, but not the mucosa samples, as indicated by 0.25 and 0.812 OOB (out of bag) error obtained, respectively (Additional File 2: Tables S2 and S3). Therefore, in the following, we mainly focus on digesta-associated microbiota. The model classified the treatments, FOS-BC and GOS-BC, quite precisely with $87.5 \%$ predicting accuracy for the digesta samples. In the digesta samples, the most important taxon that allowed discrimination of fish fed diets supplemented with BC from the other fish, was $P$. acidilactici (Fig. 6a). In the mucosa, it was the fifth most important discriminatory taxon (Fig. 6b). Both digesta (Fig. 6c) and mucosa (Fig. 6d) samples from the FOS-BC and GOS-BC diet fed fish had higher relative abundance of $P$. acidilactici compared to the FOS and control diet fed fish.

Overall results of transcriptome profiling: The RNA-seq data showed raw read counts ranging from 20.442.8 million reads with an average count of 30.1 million per sample. Uniquely mapped reads ranged 
between 15-32 million among the samples having an $71 \%$ of average unique mapping efficiency. Compared to the other treatments the GOS-BC treatment showed the highest number differently expressed genes (DEGs, Benjamini-Hochberg adjusted $p<0.1$, Table 1 ) and annotated DEGs among treatments were presented in Additional File 3. Gene ontology (GO) enrichment analysis indicated enrichments of biological process within the statistical criteria only for upregulated genes in GOS-BC vs FOS-BC and GOS-BC vs Control. The complete list of summarized GO terms generated from upregulated genes from respective comparison is available in Additional File 2: Table S4. There were no enriched biological processes for the comparisons among the other treatments due to the low number of DEGs.

Table 1

Number of differentially expressed genes (DEGs) resulted from pairwise comparisons of treatments

\begin{tabular}{|llll|}
\hline Comparisons & \multicolumn{2}{l|}{$\begin{array}{l}\text { Differentially expressed genes (DEGs) } \\
(\mathbf{q}<\mathbf{0 . 1}, \mathbf{F C}>\mathbf{1 . 5})\end{array}$} \\
\hline & Total & Upregulated & Downregulated \\
\hline FOS-BC vs FOS & 34 & 27 & 6 \\
\hline GOS-BC vs FOS-BC & 220 & 174 & 46 \\
\hline FOS vs Control & 07 & 04 & 03 \\
\hline FOS-BC vs Control & 07 & 02 & 05 \\
\hline GOS-BC vs Control & 537 & 269 & 268 \\
\hline
\end{tabular}

Overall results of global metabolome profiling: In total, 747 and 655 metabolites were detected respectively in distal intestine digesta, and blood plasma samples collected from the various treatments. The number of significantly altered metabolites among fish fed different diets are presented in the Table 2. All the detected metabolites highlighting the significantly altered metabolites in each of the comparisons between treatments are presented in the Additional File 3: Files S4 and S5 for digesta and plasma, respectively. The highest number of significantly different metabolite levels in the digesta samples were observed for the comparison between the GOS-BC and the control treatments, and the lowest between the FOS-BC and the FOS treatments i.e. 227 and 27, respectively. The metabolites showing significant differences in the GOS-BC vs control comparison indicate effects on several steps in the metabolism of most nutrients, such as amino acid and peptides, carbohydrate, fatty acid, phospholipid and sterol metabolism, nucleotide, cofactors and vitamins, as well as xenobiotics.

Regarding the FOS-BC vs FOS comparison, the few significant effects were seen generally scattered over the metabolic map, not showing clear effects on any metabolic pathway. Both FOS and FOS-BC showed quite similar number of significantly altered metabolites compared to the control. Fish fed the three supplemented diets showed high levels of metabolites linked to methylation of lysine and/or carnitine 
biosynthesis (such as N6-methyllysine, N6, N6, N6-trimethyllysine and deoxycarnitine) from the control fed fish, which did not clearly differ between fish fed the experimental diets.

Table 2

Number of significantly altered metabolites obtained from pairwise comparisons of treatments

\begin{tabular}{|lllll|}
\hline Comparisons & \multicolumn{2}{c}{$\begin{array}{l}\text { Significantly altered metabolites in } \\
\text { digesta }(\mathbf{p} \leq \mathbf{0 . 0 5})\end{array}$} & $\begin{array}{l}\text { Significantly altered metabolites in } \\
\text { plasma }(\mathbf{p} \leq \mathbf{0 . 0 5})\end{array}$ & Decreased \\
\hline $\begin{array}{l}\text { FOS-BC vs } \\
\text { FOS }\end{array}$ & 14 & Decreased & Increased & 19 \\
\hline $\begin{array}{l}\text { GOS-BC } v s \\
\text { FOS-BC }\end{array}$ & 86 & 13 & 03 & 34 \\
$\begin{array}{l}\text { FOS vs } \\
\text { Control }\end{array}$ & 60 & 23 & 18 & 48 \\
\hline $\begin{array}{l}\text { FOS-BC } v s \\
\text { Control }\end{array}$ & 63 & 56 & 104 & 60 \\
\hline $\begin{array}{l}\text { GOS-BC } v s \\
\text { Control }\end{array}$ & 165 & 63 & 65 & 101 \\
\hline
\end{tabular}

Random Forest modeling of results from digesta and plasma indicated 0.31 and $0.2500 \mathrm{~B}$ error, respectively, regarding prediction of the four treatments (Additional File 2: Tables S5 and S6, respectively). In both the digesta and the plasma, the model performed very well at classifying the control and GOS-BC treatment but encountered more difficulty at correctly distinguishing FOS group from FOS$\mathrm{BC}$ group. Although some differences were observed, many of the changes in plasma and digesta metabolites mirrored each other by dietary treatment, for example some of the metabolites important to group separation in digesta were similar to those in plasma (Additional File 1: Figure S4). Among those were metabolites important for methylation of protein lysine and/or carnitine biosynthesis (such as N6methyllysine, N6, N6, N6-trimethyllysine and deoxycarnitine) and microbiota-linked metabolism (Nmethylhydantoin).

Overall results of SCFA levels: The metabolome analyses of plasma samples did not show significant treatment effects, neither regarding the major SCFAs (acetic acid, butyric acid, and propionic acid) nor the minor (valeric acid and hexanoic acid, and branched chain fatty acids, 2-methylbutyric acid, isovaleric acid) (Additional File 2: Table S7). Regarding the digesta samples on the other hand, butyric and valeric acid showed significant treatment effects (Table 3). For both acids, significantly lower values were observed for the GOS-BC treatment compared to the control, whereas the FOS and FOS-BC showed intermediate and quite similar results. 
Table 3

SCFA concentrations in distal intestinal digesta of the fish from four treatments SCFA concentrations in digesta of distal intestine $(\mathrm{ng} / \mathrm{ml}) \mathrm{d}$

\begin{tabular}{|lllll|}
\hline & Control & FOS & FOS-BC & GOS-BC \\
\hline Acetic acid & $8.4 \mathrm{E}+04$ & $8.3 \mathrm{E}+04$ & $6.5 \mathrm{E}+04 \pm 1.8 \mathrm{E}+04$ & $1.6 \mathrm{E}+05 \pm 7.5 \mathrm{E}+04$ \\
& $\pm 3.9 \mathrm{E}+04$ & $\pm 2.6 \mathrm{E}+04$ & & \\
\hline Butyric acid & $83 \pm 11^{\mathrm{a}}$ & $68 \pm 7^{\text {ab }}$ & $60 \pm 4^{\text {ab }}$ & $54 \pm 3^{\mathrm{b}}$ \\
\hline Propionic acid & $121 \pm 17$ & $101 \pm 9$ & $87 \pm 7$ & $88 \pm 6$ \\
\hline Valeric acid & $41 \pm 4^{\mathrm{a}}$ & $34 \pm 3^{\text {ab }}$ & $32 \pm 7^{\text {ab }}$ & $28 \pm 1^{\mathrm{b}}$ \\
\hline Hexanoic acid & $254 \pm 22$ & $225 \pm 13$ & $213 \pm 1$ & $203 \pm 7$ \\
\hline 2-Methylbutyric acid & $21 \pm 4$ & $16 \pm 2$ & $16 \pm 2$ & $12 \pm 1$ \\
\hline Isobutyric acid & $25 \pm 3$ & $20 \pm 1.5$ & $21 \pm 2$ & $12 \pm 1$ \\
\hline Isovaleric acid & $13 \pm 2$ & $14 \pm 1$ & $14 \pm 1$ & \\
\hline O. Mean value \pm SEM are presented for $\mathrm{n}=8$ samples & \\
\hline Different letters indicate significant changes between treatments for SCFAs $(\mathrm{p} \leq 0.05)$.
\end{tabular}

Table 4. Composition of experimental diets for post-smolt Atlantic salmon 


\begin{tabular}{|lllll|}
\hline & \multicolumn{4}{l}{ Trial feeds (5mm pellet size) } \\
\hline Diet composition (g/ 100g) & Control & FOS & FOS-BC & GOS-BC \\
\hline Fish Meal & 15.0 & 15.0 & 15.0 & 15.0 \\
\hline Soya SPC & 11.0 & 11.0 & 11.0 & 11.0 \\
\hline Wheat Gluten & 7.2 & 8.0 & 8.0 & 8.0 \\
\hline Maize gluten & 5.0 & 5.0 & 5.0 & 5.0 \\
\hline Pea protein & 15.0 & 15.0 & 15.0 & 15.0 \\
\hline Guar Meal & 8.0 & 7.0 & 7.0 & 7.0 \\
\hline Wheat & 11.0 & 10.8 & 10.9 & 10.0 \\
\hline Fish Oil & 13.2 & 11.5 & 11.5 & 11.5 \\
\hline Rapeseed Oil & 10.4 & 11.1 & 11.1 & 11.1 \\
\hline Vit + min + AA & 4.3 & 4.9 & 4.9 & 4.9 \\
\hline Yttrium & 0.1 & 0.1 & 0.1 & 0.1 \\
\hline FOS & - & 0.1 & 0.1 & - \\
\hline Gos & - & - & - & 1.0 \\
\hline Bactocell & 28.5 & 27.9 & 27.6 & 28.1 \\
\hline Water change & 43.2 & 43.5 & 43.9 & 43 \\
\hline Analyzed moisture (\%) & -0.1 & 0.5 & 0.5 & 0.5 \\
\hline Energy (bomb calorimetry, MJ/kg) & 24.2 & 24.2 & 23.8 & 24.1 \\
\hline Crude FAT (\%) & 5.8 & 5.4 & 5.7 & 6 \\
\hline Crude protein (\%) & & & & \\
\hline
\end{tabular}

Beta glucan, nucleotides and krill were added only to the experimental diets in equal amounts.

\section{Overall results of association analysis of gut microbiota and metabolites}

The Spearman correlation analysis showed significant differences in specific microbe-metabolite correlations between the treatments. In the correlation analyses 436 digesta metabolites with the human metabolome database (HMDB) IDs were included. The circos plot and the heat map for microbemetabolite correlations in digesta samples from comparisons between FOS-BC and FOS, and GOS-BC and FOS-BC treatments are presented in Figs. 7 and 8, respectively. The circos plots indicated that carbohydrates, cofactors and vitamins, amino acids and lipids were closely correlated with genera belonging to Firmicutes, Actinobacteria and Proteobacteria phyla for both the comparisons presented. 
Heatmaps show expansion of the results shown in the circos plots. The comparison between the GOS-BC and FOS-BC treatments showed the highest number of associations between microbiota and metabolites, and most of them were significant. In the heatmaps, statistically significant results $(p<0.05)$ are indicated with asterisks. Correlations values $(R)$ and $p$-values for the specific microbe-metabolite correlations are presented in Additional File 4.

Supervised multivariate analysis on the combined data matrix of microbiota (at genus level) and metabolome and in the digesta with the OPLS-DA method pointed out clearer separation between GOS$\mathrm{BC}$ and FOS-BC treatments than between FOS-BC and FOS treatments as indicated by the first component (Additional File 1: Figure S5). Variable importance plot (not shown) based on the OPLS-DA model was used to identify differential microbes and metabolites contributing to the separation of one group compared to the other (Variable Importance on Projection, VIP values $>1$ and correlation coefficients $p<0.05$ ). The list of microbiota and metabolites fulfilling the above statistical criteria in each comparison between dietary treatments are presented in the Additional File 5. Genus Pediococcus was identified as an important variable in both FOS-BC and GOS-BC treatments from the control and the FOS treatment.

\section{Comparison of the FOS and the FOS-BC treatment}

As mentioned above, the fish in the FOS and the FOS-BC treatments showed no significant difference in growth rate, FCR or histological appearance of the pyloric caeca and distal intestine. The results of metabolome analyses of digesta and plasma indicated very few significant alterations among the more than 750 identified metabolites, indicating no important metabolic differences between fish fed these two diets.

Regarding the microbiota results the FOS and FOS-BC treatments did not show significantly different alpha diversity, neither in digesta (Additional File 1: Figures S2a - S2b) nor mucosa samples (Additional File 1: Figures S2c - S2d). On the other hand, beta-diversity from the FOS-BC treatment showed clear separation from those in the FOS treatment for the digesta samples $(p=0.009$, Figure $4 a)$, but not for the mucosa samples (Figure 4b). The number of differentially abundant genera was 19, and 15 of them showed highest abundance in the FOS-BC fed fish. Pediococcus and Staphylococcus were among the genera showing increase. The complete list of genera in digesta which showed significant changes in their abundance in FOS-BC group compared to the FOS group is shown in Additional File 2: Table S1. Further, fish fed the FOS-BC diet had higher relative abundance of $P$. acidilactici compared to the fish fed the FOS diet in both digesta and mucosa samples (Figure $6 \mathrm{c}$ and $6 \mathrm{~d}$ ).

The global transcriptomic changes in the distal intestine of fish fed the FOS-BC diet compared to those fed the FOS diet showed a low number of DEGs (27 up- and 6 down-regulated, Table 1, list of differentially expressed annotated genes in Additional File 3: File S1). Results of G0 enrichment analysis did not indicate enrichments of biological processes within the statistical criteria for particular comparison due to the low number of DEGs. 
Regarding the results of association analysis of gut microbiota and metabolite, Circos plots showed that 4 different classes of metabolites, carbohydrates, cofactors and vitamins, amino acids and lipids were closely correlated with genera belonging to Firmicutes, Actinobacteria, Proteobacteria and Epsilonbacteoeota phyla (Figure 7a). Similar observations were made for the metabolites in plasma (data not shown). As shown in the heatmap (Figure 8a), the 15 genera showing increase in the FOS-BC treatment compared to FOS treatment showed positive correlation with the $10-11$ significantly changed metabolites in the respective comparison. Some of these correlations were statistically significant $(\mathrm{p}<$ 0.05). Genus Pediococcus showed positive and significant associations with 10 metabolites including lactose, ergosterol, chiro-inositol and ribose (Additional File 4: File S1).

Supervised multivariate analysis on the combined data matrix of microbiota and metabolome in the digesta pointed out less clear separation between FOS-BC and FOS treatments (Additional File 1: Figure S5). The FOS-BC treatment showed 41 separating factors from the FOS treatment (Additional File 5: File S1). The genus Pediococcus was identified as an important variable in FOS-BC group distinguishing it from the FOS group.

\section{Comparison of the FOS-BC and the GOS-BC treatment}

As mentioned above, the fish in the FOS-BC and the GOS-BC treatments showed no significant difference in TGC, FCR or histological appearance of the pyloric caeca and distal intestine.

Regarding microbiota profiling of the digesta samples the results showed that replacement of GOS for FOS in the FOS-BC diet caused a significant decrease in alpha diversity (observed species, $p=0.046$ and Shannon index, p-value $=0.0046$ ) (Additional File 1: Figures S2a - S2b), although the mucosa samples did not show significant diet effects on alpha diversity (Additional File 1: Figures S2c-S2d).

Beta diversity in the digesta samples, revealed that the microbiota in fish from the GOS-BC treatment clustered close to, but distinct from that of the FOS-BC treatment ( $p=0.02$, Figure $4 a)$. The mucosa samples (Figures $4 b$ ) did not show significant differences in beta diversity among the samples.

Fish fed the GOS-BC diet, compared to those fed the FOS-BC diet, showed reduction in 24 genera including Kurthia, Savagea, Staphylococcus, Vagococcus and Peptostreptococcus (Additional File 2: Table S1). Moreover, both digesta and mucosa samples from the fish fed the GOS-BC diet had higher relative abundance of $P$. acidilactici compared to the fish fed the FOS-BC diet (Figure $6 \mathrm{c}$ and $6 \mathrm{~d}$ ).

Global transcriptome analyses showed major differences in the distal intestinal tissue between fish fed the GOS-BC diet and FOS-BC diet. In the fish fed GOS-BC diet 220 genes were differentially expressed, 174 up- and 46 down-regulated compared to the fish fed FOS-BC diet (Table 1, Additional File 3: File S2). Among the upregulated genes in fish fed with GOS-BC diet were cysteine knot cytokine members, interleukin 17 and receptors, $/ / 17 a, i / 17 a / f 1$ and $i 17 r a$; TNF superfamily members and receptors tnfrsf $1 b$, tnfrsf1, tnfrsf9a and tnfsf18; beta trefoil cytokine family member il-1rl; and a number of chemokines (Additional File 3: File S2). The fish in the GOS-BC treatment also showed an increase in expression of 
transcripts of NADPH oxidases family of enzymes, dual oxidases (duox and duox2) and NADPH oxidase activator 1 (noxa1a and noxo1b) and key antioxidant enzyme, glutathione peroxidase 1b (gpx1b).

The GO enrichment analysis was performed for the genes upregulated in the GOS-BC treatment compared to the FOS-BC treatment. The summarized GO terms generated from enriched nonredundant biological function GO terms are presented in Figure 9 for the upregulated genes. The complete list of non-redundant GO terms generated from upregulated genes is available in Additional File 2: Table S4. Among the enriched $\mathrm{GO}$ biological process terms were immune response, apoptotic process, inflammatory response, response to stress and reactive oxygen species metabolic process.

The global metabolome profiling showed that replacement of FOS in the FOS-BC diet with GOS significantly altered a high number of metabolites in both digesta and plasma (Table 2, Additional File 3: Files S4 and S5 respectively for digesta and plasma). Unique for the GOS-BC treatment were high levels of long chain saturated, long chain monounsaturated, long chain polyunsaturated as well as branched fatty acids, most pronounced for digesta (Additional File 3: File S4). Among those metabolites were n-3 (EPA, 20:5n-3 and docosapentaenoic acid, DPA, 22:5n-3) and n-6 fatty acids (linoleic acid; $18: 2 n-6$, eicosadienoic acid, 20:2n-6, arachidonic acid, 20:4n-6, adrenic acid, 22:4n-6 and dihomo-gamma-linolenic acid; 20:3n-6, DPA, 22:5n-6 and tetracosahexaenoic acid, 24:6n-3). The GOS-BC fed fish also showed increased levels in the digesta of acetylcarnitine, propionylcarnitine, butyrylcarnitine compared to FOS-BC fed fish, as well as compared to the other treatments. Levels of several sphingomyelins, ceramides and hexosylceramides were also increased distinctively in the GOS-BC fed fish compared to the FOS-BC fed fish and fish from the other treatments.

No significant changes in SCFA levels in plasma or digesta were observed in the comparison between GOS-BC and FOS-BC treatments (Additional File 2: Table S7, and Table 3 for plasma and digesta samples respectively).

The association analysis of gut microbiota and metabolite presented in the Circos plot showed that seven different classes of metabolites including nucleotides, carbohydrates, peptides, cofactors and vitamins, xenobiotics, amino acids and lipids were closely correlated with genera mainly belonging to Firmicutes, Actinobacteria and Proteobacteria phyla (Figure 7b). Similar observations were made for the metabolites in plasma (data not shown). All the 24 genera showing decrease in GOS-BC treatment compared to FOS-BC treatment displayed positive correlation with $54-56$ metabolites (Figure 8b). Some of these correlations were statistically significant $(p<0.05)$. Negative correlations were observed between significantly increased metabolites including $n-3$ and $n-6$ polyunsaturated fatty acids and the altered bacterial genera in GOS-BC treatment compared to the FOS-BC treatment (Additional File 4: File S2).

Supervised multivariate analysis on the combined data matrix of microbiota and metabolome in the digesta with the OPLS-DA method showed a clear separation between GOS-BC and FOS-BC treatments as indicated by the first component (Additional File 1: Figure S5). Variable importance plot identified showed 115 separating factors in the GOS-BC group compared to the FOS-BC treatment (Additional File 5: File S2). 


\section{Discussion}

\section{Effects of supplementation with P. acidilactici to the FOS diet}

We observed no significant effect of supplementing $P$. acidilactici to the FOS diet on TGC, FCR or histological appearance of the pyloric caeca and distal intestine. Improved growth was observed for fish fed the FOS and P. acidilactici diet when compared to the commercial control diet. However, it is not possible for us to evaluate whether the synbiotic treatment was the causative factor for the observed improvements in growth, since the experimental diets also contained elevated levels of vitamin $C$ and $E$, beta glucan and nucleotides, and had a partial substitution of standard fish meal with krill meal. A previous study reported no significant change in growth when Atlantic salmon were fed a diet supplemented with the same synbiotic combination [7]. As such, it is possible that the improved growth observed in the current study was caused by other dietary supplements besides the synbiotics, or by a combined effect.

The observation in the present study showing that alteration in diet composition, i.e. supplementation with $P$. acidilactici to the FOS containing diet, modified the digesta-associated microbiota in the distal intestine of post-smolt Atlantic salmon is in line with other recent observations in salmon [25]. The same is the case for the results regarding the mucosa-associated microbiota, which showed resistance towards dietary changes, again confirming the results from Li et al [25] and supported by the findings from Abid et al [7]. Moreover, our findings that, irrespective of diet, the phyla Firmicutes and Proteobacteria and genera Lactobacillus and Leuconostoc were predominantly present in the digesta are also strengthening previous findings which indicate that these bacterial groups are among the core digesta-associated microbiota in post-smolt Atlantic salmon [25-27]. In more detail, alpha diversity or species richness of the digesta-associated microbiota in the fish fed FOS-BC diet did not differ significantly from those fed FOS diet indicating that supplementation of $P$. acidilactici to the FOS diet did not alter the number of bacterial species in digesta. Similar to the present observation, a previous study [7] has reported that dietary application of FOS and $P$. acidilactici did not induce significant changes in alpha diversity in the digesta-associated microbiota in the distal intestine of Atlantic salmon. On the other hand, the fact that fish fed FOS-BC diet showed a significantly different beta diversity in the digesta from that of the fish fed FOS diet, indicates an ability of $P$. acidilactici when in combination with FOS to modulate the bacterial composition by altering abundance of the various bacteria. As expected, $P$. acidilactici showed relatively higher abundance in both the digesta and mucosa samples of fish fed FOS-BC diet compared to those fed FOS diet, suggesting strengthened establishment in the gut. Previous studies have demonstrated the ability of $P$. acidilactici to populate the distal intestine of Atlantic salmon when it is used as dietary supplementation [10] or in combination with FOS [7]. In the present study, P. acidilactici was found to be the key factor separating gut microbiota of fish fed FOS-BC diet from that of the fish fed FOS diet indicating its importance for modulating gut microbiota profile. The $P$. acidilactici was also the key factor for distinguishing the digesta-associated microbiota profiles of FOS-BC fed fish from those in the fish fed control diet. 
Since gut microbiota plays an important role in shaping the fecal metabolome, we expected that the combined evaluation of microbiota and metabolomics data could give us a better understanding of the possible functional implications of the diets used in the present study. We analyzed the global metabolite signature and SCFA levels in both digesta and blood plasma, expecting that microbiota and metabolic interactions may give local as well as systemic effects [28, 29]. However, only a few metabolites changed significantly between the fish fed FOS-BC and FOS diets, demonstrating that the supplementation of $P$. acidilactici to the FOS diet had a surprisingly low impact on the metabolome of both the gut content and the systemic circulation. As a consequence, interactions between gut microbiota and metabolites were also few. The general lack of strong host responses after $P$. acidilactici supplementation to the FOS diet was also observed on the transcriptional level.

\section{Effects of replacement of GOS for FOS in the FOS-BC diet}

No previous published studies have compared GOS-BC supplementation with FOS-BC supplementation to fish feeds. However, supplementation of GOS-BC to a control diet was previously reported to increase growth performance and lower FCR in rainbow trout fingerlings [30] and juvenile rockfish, Sebastes schlegeli [6]. In contrast to those observations, we observed no change in growth performance between fish fed the GOS-BC and the control diet in the present work. Nevertheless, a resent study also reported no significant changes in growth parameters in rainbow trout [19] with the application of same synbiotic combination.

Effects on Microbiota: Regarding the effects on the digesta microbiota, the effects of replacing FOS with GOS comprised a reduction in alpha diversity i.e. a decrease in the number of species present. Also, beta diversity was altered indicating a shift of abundance of some of the bacteria. Fish in the GOS-BC treatment showed increased relative abundance of $P$. acidilactici compared to the FOS-BC treatment. This increase in both the digesta and mucosa indicates enhanced establishment of $P$. acidilactici when in combination with GOS relative to FOS. Increased abundance of $P$. acidilactici was also reported in Rainbow trout treated with GOS and the same probiotic species [19]. P. acidilactici strains are known to produce bacteriocins, pediocins, which may exert antagonistic effects towards a variety of bacteria including both gram negative and positive species [31, 32]. Therefore, decreased alpha diversity and the reduced abundance of several genera observed when replacing FOS with GOS in the diet could potentially be a result of increased antagonistic effects exerted by $P$. acidilactici when combined with GOS. This was further supported by the observation that the GOS-BC diet also reduced the abundance of more genera in the digesta than FOS-BC, relative to the control diet.

Impact on the metabolome of digesta and blood plasma. Replacing GOS with FOS increased levels of short, medium, and long chain acyl-carnitines in both digesta and plasma. This suggests that GOS could directly influence or act as a substrate for the gut microbiota to supply the intestinal mucosa and the body with compounds having important functions in lipid transport and metabolism. Carnitine and its acyl esters (acyl-carnitines) are essential for transport of fatty acids across the outer and inner mitochondrial membranes, for the mitochondrial beta-oxidation of long-chain fatty acids, as well as 
for maintenance of the ratio of acetyl-CoA/CoA $[33,34]$. On the other hand, gut bacteria can utilize carnitine for protection against osmotic stress [35].

The increase in several sphingolipids, including sphingomyelin and interrelated products such as ceramide, and hexosylceramides in the fish fed GOS-BC diet suggests that GOS may affect and possibly improve various barrier functions. Sphingolipids, mainly ceramide, act as signaling molecules and are involved in diverse processes including epithelial integrity, cell growth and death, apoptosis, immunity and inflammation $[36,37]$. As they are important in orchestration of immune responses (cytokine release, inflammatory responses and initiation of apoptosis of the infected cell) and eliminating invading pathogens [37], many pathogens have developed strategies to exploit host cell sphingolipid pathways to change the sphingolipid balance to facilitate their colonization [38]. Therefore, it is possible that the increase in sphingolipid levels in GOS-BC fed fish might trigger an immune response. The transcriptome results seem to indicate such an effect as explained later.

Most of the SCFAs levels were significantly similar among the four treatments except butyric acid and valeric acid which showed a reduction in fish fed GOS-BC diet compared to those fed control. SCFAs are among the most important gut microbial products and are reported to exert multiple beneficial effects on vertebrates by involvement in energy homeostasis and healthy immune responses [39, 40]. However, a few studies investigating pre- pro- or synbiotic applications in fish have reported effects on SCFA production and the observations were quite different from ours. For example dietary application of Enterococcus faecalis in Javanese carp, Puntius gonionotus increased the intestinal propionic and butyric acid, but not acetic acid [41]; and Alcaligenes sp. increased intestinal acetic acid, but not butyric acid levels in Malaysian Mahseer, Tor tambroides [42]. Mammalian studies have indicated that formation of SCFAs by intestinal bacteria is regulated through many different host, environmental, dietary and microbiological factors with substrate availability, microbial species composition and intestinal transit time playing a larger role [43]. Therefore, relatively similar SCFA concentrations observed among fish in the three treatments as well as controls possibly could indicate relatively stable environmental and host factors rather than to the different dietary and microbiological factors. However, this needs to be further investigated.

Impact on the transcriptome. The observed transcriptomic changes upon the FOS to GOS exchange, i.e. upregulation of genes coding for a number of cytokines and/or their receptors (I/17a, i/17a/f1, i17ra, tnfrsf1b, tnfrsf1, tnfrsf9a, tnfsf18 and il-1rl) indicate alterations in communication between innate and adaptive immune systems $[44,45]$. The increase in expression of the toll-like receptor 18 gene $(\operatorname{tr} 18)$, important for bacterial pathogen recognition [46], and of the antibacterial peptide gene (hepc1) may indicate effects of the exchange of prebiotic on immune functions important for disease resistance. The same regards the increase in expression of transcripts (duox, duox2, noxa1a and noxo $1 b$ ) important for reactive oxygen species generation and innate host defense pathways on mucosal surfaces, cellular signaling, regulation of gene expression and cell differentiation $[47,48]$. The GOS-BC treatment also displayed increased expression of the key antioxidant enzyme, gpx $1 b$, involved in protection of the fish from oxidative stress. 
Upregulation of genes involved in immune and other defense mechanisms does not necessarily mean increased resistance towards infection diseases or other stressors - it could also representant a natural adaptation to the diet. This also can be possibly due to the increase production of pediocin with antimicrobial properties by highly abundant $P$. acidilactici when in combination with GOS. Activation of defense mechanisms may also be a sign of inflammatory responses. However, the histological appearance of the distal intestine did not indicate altered state of inflammation which was evaluated as mild to moderate for all treatments. The mechanism underlying the alteration in the transcriptome may be the combined effects of a) the direct influence of GOS, and b) the indirect influence caused by the action of microbiota on the GOS and c) effects of altered metabolite production in the microbiota linked to the alteration in beta diversity. Support for the suggestion of beneficial effect of GOS on disease resistance is found in studies with rainbow trout in which a combination of GOS and $P$. acidilactici increased antioxidant defense, innate immune response and resistance to streptococcosis $[17,18]$.

Correlations between impacts on microbiota, metabolome and transcriptome. Replacing FOS with GOS in the FOS-BC diet showed significant impacts on gut microbiota and metabolite interactions. Spearman correlation analysis revealed that metabolites including nucleotides, carbohydrates, peptides, cofactors and vitamins, xenobiotics, amino acids and lipids were closely correlated with genera mainly belonging to Firmicutes, Actinobacteria and Proteobacteria phyla. Previous studies in fish and mammals have reported the involvement of gut microbiota in lipid metabolism and energy homeostasis $[49,50]$ and de novo synthesis of essential amino acids and vitamins [51, 52]. This suggests that supplementation of GOS and $P$. acidilactici in the diet could have modulated gut microbiota associated with some of those functions in the post-smolt Atlantic salmon in the present study as well. Further, increased transcripts and metabolite levels related to immunomodulatory effects could also potentially link to the increased abundance of $P$. acidilactici when in combination with GOS.

\section{Conclusions}

This study reports effects on growth performance, gut health, microbiome, transcriptome, metabolome and their interactions in post-smolt Atlantic salmon when supplementation of a diet containing the prebiotic FOS with the probiotic $P$. acidilactici and exchanging FOS with GOS in a diet containing $P$. acidilactici. No significant effects of these dietary alterations were detected on growth or histomorphological appearance of the gut. Supplementation with $P$. acidilactici to the FOS containing diet altered digesta associated microbiota to some degree, whereas the mucosa-associated microbiota seemed relatively resistant to such dietary modulation. This probiotic also induced moderate effects in some of the assessed components of the metabolome and transcriptome. Replacing FOS with GOS in FOS-BC diet induced several, clear effects on many of the observed biomarkers which may indicate that GOS induces important effects on the microbiota, metabolome in the digesta as well as the endogenous metabolism, as well as on the mucosal metabolism and function. However, those alterations did not significantly impact the growth performance of GOS-BC group. Further infection challenge and stress studies are needed to ascertain the efficacy of dietary application of GOS and P. acidilactici along with 
functional ingredient mixes as an immune stimulant strategy against disease outbreaks and stressful events.

\section{Materials And Methods}

Experimental design, study parameters and analytical procedures used to evaluate the effect of functional seawater transfer diets for Atlantic salmon are illustrated in the Figure 1 and explained in the subsequent sections.

\section{Feeding trial}

A sea water feeding trial was conducted with post smolts Atlantic salmon at LetSea research facility in Dønna, Norway from 29/05/2018 to 16/09/2018, following the Norwegian laws regulating the experimentation with live animals.

Atlantic salmon with average weight $172 \pm$ SEM $0.89 \mathrm{~g}$ were randomly assigned to 16 net pens $(5 \times 5 \times 5 \mathrm{~m})$ with 300 fish each. Four feeds were prepared by Biomar AS, a control diet based on standard grower feed recipes and three experimental diets. The experimental diets contained elevated vitamin $\mathrm{C}$ and $\mathrm{E}$, beta glucan and nucleotides, and had a partial substitution of standard fish meal with krill meal. The experimental diets were further supplemented with either; prebiotic fructo-oligosaccharide (FOS, $0.1 \%$ ), FOS (0.1\%) and Bactocell (0.03\%) (FOS-BC); or galacto-oligosaccharide (1.0\%) and Bactocell $(0.03 \%)$ (GOS-BC) (Table 4). Bactocell (Lallemand Inc., Cardiff, UK) is authorized by the European Union for the use in fish and shrimp [53] and has already been used in salmon fry and freshwater stage diets. All feeds were produced at Biomar Feed technology Center in Brande, Denmark. Four randomly distributed pens were allocated for each dietary group. Fish were fed above mentioned four feeds: acclimatization diets (3,5 $\mathrm{mm}$ pellets) during the first 5 weeks following seawater transfer, and then the trial diets $(5 \mathrm{~mm}$ pellets) for 10 additional weeks. Bulk weights for each pen were registered at the end of the acclimatization period and the 10-week feeding trial period to determine start and end weight of the experimental fish. During the experimental period, average seawater temperature of $12.4 \pm 1.8^{\circ} \mathrm{C}$, salinity of $31.9 \pm 0.7 \mathrm{ppt}$ and oxygen of $10.0 \pm 1.1 \mathrm{mg} / \mathrm{l}$ were reported.

At the end of the feeding trial, four fish were randomly taken from each net pen, anesthetized with tricaine methanesulfonate (MS222 ${ }^{\circledR}$; Argent Chemical Laboratories, Redmond, WA, USA), weighed individually and euthanized by a sharp blow to the head. Blood samples were drawn from the caudal vein using heparinized syringes and placed on ice before plasma collection. Plasma was collected after centrifugation at $2000 \mathrm{~g}$ for $10 \mathrm{~min}\left(4^{\circ} \mathrm{C}\right)$ and snap frozen in liquid $\mathrm{N} 2$. After cleaning the exterior of each fish with $70 \%$ ethanol, the distal intestine was aseptically removed, opened longitudinally and digesta was collected into a $50 \mathrm{ml}$ sterile centrifuge tube. The digesta was mixed thoroughly with a spatula and aliquots were transferred into $1.5 \mathrm{ml}$ sterile Eppendorf tubes and snap frozen in liquid $\mathrm{N}_{2}$ and stored at $-80^{\circ} \mathrm{C}$ for the analysis of the digesta-associated intestinal microbiota and metabolomic profiling. The mid-section of the same distal intestine was excised and rinsed 3 times in sterile phosphate-buffered 
saline. Subsequently, the tissue was transversely divided into 3 pieces, respectively, for histological evaluation (fixed in 4\% phosphate-buffered formaldehyde solution for $24 \mathrm{~h}$ and transferred to $70 \%$ ethanol for storage), RNA-Sequencing (preserved in RNAlater solution and stored at $-20^{\circ} \mathrm{C}$ ) and mucosaassociated intestinal microbiota analysis (snap frozen in liquid $\mathrm{N}_{2}$ and stored at $-80^{\circ} \mathrm{C}$ ).

The performance of the fish in each dietary group was calculated using the thermal growth coefficient and specific growth rate, which are considered as good predictors of salmon growth [54]. Statistical analysis of growth parameters among the treatments was performed by one-way ANOVA after checking the fulfillment of all the pertinent assumptions, normality of the distribution and homogeneity of variances. Pairwise comparisons were analyzed using Tukey's honestly significant different (HSD) test, and $p \leq 0.05$ was considered as statistically significant.

\section{Histological analysis}

The gut tissue sections (total of 64 fish, $n=16$ per dietary group, $n=4$ fish randomly selected from each of the 4 pens allocated for a dietary group) of pyloric caeca and distal intestine were evaluated by light microscopy with focus on the characteristic morphological changes of soybean meal-induced enteritis (SBMIE) in Atlantic salmon distal intestine, that consist of shortening of mucosal fold length, increase in width and inflammatory cell infiltration of the submucosa and lamina propria, and reduction in enterocyte supranuclear vacuolization. Additionally, for the pyloric caeca, changes in the vacuolization of the intestinal epithelial cells were evaluated. Normally, little to no vacuolization is present in the intestinal epithelial cells of the pyloric caeca and mid intestine. Increased vacuolization (or hyper-vacuolization) is observed in fish affected by the so-called lipid malabsorption syndrome (LMS) that manifests in its advanced form as 'floating feces' (steatorrhea).

The degree of change was graded using a scoring system with a scale of 0 - 4 where 0 represented normal; 1 , mild; 2 , moderate; 3 , marked, and 4 , severe changes. The histological evaluation was conducted randomly and blind and assignment of individual samples to the treatments was obtained after the evaluation was completed.

Differences in histological scores for the evaluated morphological characteristics of the intestinal tissue were analyzed for statistical significance using ordinal logistic regression run in the $\mathrm{R}$ statistical package (version 3.6.3; 2020) within the RStudio interphase (version 1.3.1093; 2020). Differences were examined based on odds ratios of the different treatments having different histology scores compared to the reference diet. Control was used as the reference.

\section{Microbiota analysis}

DNA extraction: For analysis of the distal intestinal microbiota, a total of 32 fish samples were used. Two fish were randomly selected from each of the 4 pens allocated for a dieatary group to have $n=8$ fish per diatary group. The DNA was extracted from respective digesta and mucosa samples following the protocol of QIAamp Fast DNA Stool Kit (Qiagen, Crawley, UK) with some modification as suggested by 
Knudsen, et al. [55]. Samples were pre-processed with a bead-beating protocol of three times in the Fastprep at $6.5 \mathrm{~m} / \mathrm{s}$ for $30 \mathrm{~s}$ with a mix of beads $(120 \mathrm{mg}$ acid-washed glass beads $(150-212 \mu \mathrm{m})$ and $240 \mathrm{mg}$ Zirconium oxide beads $(1.4 \mathrm{~mm})$ ). For quality control of the microbiota profiling protocol, along with the each of the DNA extraction batch, two 'blanks' (without any sampling materials) and two 'positive controls' i.e. mock (microbial community standard from Zymo-BIOMICS ${ }^{\mathrm{T}}{ }^{\text {, }}$, Zymo Research, California, USA) were included. The mock contains 8 bacteria (Pseudomonas aeruginosa, Escherichia coli, Salmonella enterica, Lactobacillus fermentum, Enterococcus faecalis, Staphylococcus aureus, Listeria monocytogenes, Bacillus subtilis) and 2 yeasts (Saccharomyces cerevisiae, Cryptococcus neoformans).

PCR amplification of V1-V2 region of the 16S rRNA gene. PCR amplification was carried out using 27F (5' AGAGTTTGATCMTGGCTCAG 3'), and 338R-I (5' GCWGCC TCCCGTAGGAGT 3') and 338R-II (5'

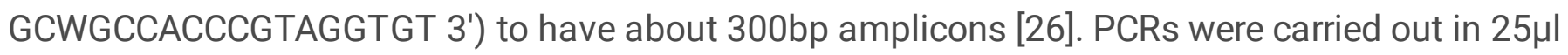
reactions with $12.5 \mu \mathrm{l}$ of Phusion® HighFidelity PCR Master Mix (Thermo Scientific, CA, USA); $1 \mu \mathrm{M}$ of forward and reverse primers, and $1 \mu$ template DNA. Undiluted and 1:2 diluted templates were used, respectively, from the digesta and mucosa. The PCR conditions were as follows: initial denaturation at $98 \bowtie \mathrm{C}$ for $7 \mathrm{~min}$ followed by initial 10 cycles with denaturation at $98^{\circ} \mathrm{C}$ for $30 \mathrm{~s}$, annealing temperature decreasing from $63^{\circ} \mathrm{C}$ to $53^{\circ} \mathrm{C}$ for $30 \mathrm{~s}$ at each temperature and extension at 72 for $30 \mathrm{~s}$; followed by 25 further cycles with denaturation at $98{ }^{\circ} \mathrm{C}$ for $30 \mathrm{~s}$, annealing at $53{ }^{\circ} \mathrm{C}$ for $30 \mathrm{~s}$, and extension at $72{ }^{\circ} \mathrm{C}$ for $30 \mathrm{~s}$; followed by a final extension at $72{ }^{\circ} \mathrm{C}$ for $10 \mathrm{~min}$. Negative PCR controls were included by replacing the template DNA with molecular grade water. PCR was performed in duplicate, pooled, and examined by $1.5 \%$ agarose gel electrophoresis.

Library preparation and sequencing. Library preparation of the products from amplicon PCR was performed using the Quick-16 ${ }^{\text {TM }}$ NGS Library Prep Kit (Zymo Research) following the instructions from the producer. Briefly, PCR products were first enzymatically cleaned up followed by a PCR to add barcodes. Subsequently, the libraries were quantified by qPCR, pooled, and purified. A representative number of individual libraries were evaluated for DNA quality in Agilent Bioanalyzer 2100 system (Agilent Technologies, California, USA). The final pooled library was then denatured and diluted to $8 \mathrm{pM}$ and sequenced on Illumina MiSeq platform with Miseq Reagent Kit v3 (600-cycle) (Illumina) to generate paired-end read. $20 \%$ of 8 pM PhiX control was added as an internal control.

Bacterial DNA quantification by $q P C R$. As an extra measure to identify contaminating sequences, qPCR was performed to quantity 16S rRNA gene in the diluted DNA templates (samples, blanks, and mocks) used for the amplicon PCR. The qPCR assays were performed using a universal primer set (forward, 5'CCA TGA AGT CGG AAT CGC TAG-3'; reverse, 5'-GCT TGA CGGGCG GTG T-3') as described previously [56, 57]. The qPCR was performed using the LightCycler 96 (Roche Applied Science, Basel, Switzerland) in a $10 \mu \mathrm{l}$ reaction volume; $2 \mu \mathrm{l}$ of PCR-grade water, $1 \mu \mathrm{l}$ diluted DNA template, $5 \mu$ l LightCycler 480 SYBR Green I Master Mix (Roche Applied Science) and $1 \mu \mathrm{l}(3 \mu \mathrm{M})$ of each primer. The qPCR program used as follows; an initial enzyme activation step at $95^{\circ} \mathrm{C}$ for $2 \mathrm{~min}, 45$ three-step cycles of $95^{\circ} \mathrm{C}$ for $10 \mathrm{~s}, 60^{\circ} \mathrm{C}$ for $30 \mathrm{~s}$ and $72{ }^{\circ} \mathrm{C}$ for $15 \mathrm{~s}$, and a melting curve analysis at the end. Quantification cycle (Cq) values were 
determined using the second derivative method [58] and bacterial DNA standards were used as inter-plate calibrators and the inter-plate calibration factor was calculated as described previously [59].

Bioinformatics analysis of microbiome sequencing data: This was performed using QIIME2 version 2 [60, 61]. The demultiplexed paired-ended reads were denoised, trimmed and quality filtered using the DADA2 algorithm [62] in QIIME2. Primer sequences were trimmed off (forward reads, first 20bps; reverse reads, first 18bps) and the reads were truncated at the position where the median Phred quality crashed (forward reads, at position $290 \mathrm{bp}$; reverse reads, at position $238 \mathrm{bp}$ ) and low-quality reads were filtered out. Chimeric sequences were removed after merging the reads. The taxonomy was assigned to resulting amplicon sequence variants (ASVs) tables by a Scikitlearn Naive Bayes machine-learning classifier [63], which was trained on the SILVA 132 99\% ASVs [64] that were trimmed to exclusively include the regions of 16S rRNA gene amplified by the primers used in the current study. ASVs assigned as chloroplast and mitochondria were removed from ASVs table. The ASV table was then filtered to remove ASVs that are without a phylum-level taxonomic assignment or appeared in only one biological sample. Low abundance ASVs with total abundance of less than 2 across all the samples were also filtered out. Contaminant sequences were detected using control samples (negative PCR reactions, DNA extraction blanks and mocks) and bacterial DNA quantification data obtained from qPCR mentioned in the previous section, as suggested by Davies et al. [65]. In general contaminants are frequently found in negative controls and blanks and show a negative correlation with the bacterial DNA concentration. Moreover, contaminants also can be foreign ASVs in mocks those are not belonging to the original included bacteria. In total 19 and 11 ASVs were removed from mucosa and digesta samples respectively based on their presence in mocks, extraction blanks and negative PCR controls, and their negative correlation with bacterial DNA concentration. The ASVs removed from mucosa samples belonged to the genera Rhodoluna, Cutibacterium, Flavobacterium, Afipia, Curvibacter, Limnohabitans, Polynucleobacter, Ralstonia, Undibacterium, Pseudomonas, Aliivibrio and Brevinema. On the other hand, the removed contaminants from digesta samples belonged to the genera Cutibacterium, Flavobacterium, Curvibacter, Undibacterium and uncultured bacterium from order Saccharimonadales. After filtering, a total number of 1358 and 736 ASVs were obtained for digesta and mucosa samples, respectively. The ASVs filtered from the raw ASV table were also removed from the representative sequences.

To compute alpha and beta diversity indices, the ASV tables were rarified at 28300 and 1833 reads for digesta and mucosa samples respectively in order to have an even number of reads across all the samples. Alpha diversity was calculated using observed species and Shannon`s diversity indices. Beta diversity was evaluated using Bray-Curtis. QIIME2 and MicrobiomeAnalyst package $[66,67]$ was used to analyze abundant taxa among treatments, alpha diversity (Kruskal-Wallis), beta diversity (PERMANOVA test), Random forest analysis and graphical presentations of data using ASV table at species level.

\section{Global transcriptomic profiling}

RNA Sequencing. Total RNA was extracted from distal intestinal digesta of 28 fishes ( $\mathrm{n}=7$ per dietary group) from the 32 fishes used for microbiota analysis using Invitrogen PureLink RNA Mini Kit with 
column based purification (Thermo Fisher Scientific, Waltham, USA ), following the manufacturer's protocol. Tissues were homogenized twice at $5000 \mathrm{xg}$ for $15 \mathrm{~s}$ with zirconium oxide beads $(1.4 \mathrm{~mm})$ using FastPrep-24 ${ }^{\mathrm{TM}}$ (MP Biomedicals, Thermo Fisher Scientific, Waltham, USA). RNA integrity was checked using an Agilent 2200 TapeStation (Agilent Technologies, Santa Clara, USA), and RNA quantity and RNA purity were measured using Epoch Microplate Spectrophotometer (BioTeK Instruments, Winooski, USA).

Library preparation and RNA sequencing was performed by Norwegian National Sequencing Center (Oslo Norway). Libraries were prepared using TruSeq ${ }^{\circledR}$ Stranded mRNA Library Prep kit with TruSeq RNA unique dual indexes in accordance with the manufacturer's protocol (Illumina, San Diego, USA). Sequencing was performed on the Illumina SP Novaseq flow cell to yield $100 \mathrm{bp}$ single end reads.

Bioinformatics analysis of RNA-Seq data: After demultiplexing raw sequencing data were processed for quality and adapter trimming using Cutadapt [68] with -q 25, 20, quality-base $=33$, trim-n -m 20 parameters, followed by a further quality check with FastQC (https://www.bioinformatics.babraham.ac.uk/projects/fastqc/). Quality trimmed reads were mapped to the indexed Atlantic salmon genome, ICSASG v2 with refseq genes using HISAT2 package [69] in Norwegian e-Infrastructure for Life Sciences (NeLS) galaxy platform developed by ELIXIR Norway [70]. HTSeq [71] was used to compute gene expression values. Differentially expressed genes in fish fed with functional diets in comparison to their control diet fed counterparts were determined using DESeq2 [72] and the differences were considered statistically significant when the adjusted $p$-value $(q)$ with the Benjamini-Hochberg procedure $\leq 0.1$.

Functional annotation and gene ontology analysis of DEGs: Functional annotation of the DEGs was performed using g:Profiler online tool $[73,74]$ and manually inspecting the Ensembl (http://www.ensembl.org) and NCBI (https://www.ncbi.nlm.nih.gov/) data bases. Gene ontology enrichment analysis (GO) was carried out also with g:Profiler online tool. For the calculation of statistically significant enrichment, all the known genes of the Atlantic salmon in the Ensembl database (Ensembl 100, Ensemble genome 47) were considered and the threshold to determine GO terms was set as Benjamini-Hochberg FDR (False Discovery Rate) value of 0.1. Enriched GO terms were then summarized by removing redundant $\mathrm{GO}$ terms and visualized in semantic similarity-based scatterplots using REVIGO online tool [75].

\section{Short chain fatty acids and metabolites analysis}

Targeted short chain fatty acids analysis and global untargeted metabolite profiling were performed by Metabolon, Inc. (Morrisville, USA). Plasma and digesta collected from the same 32 fishes ( $n=8$ per dietary group) were used for microbiota and transcriptomics analysis.

SCFA analysis. For the SCFA analysis, samples were spiked with stable labelled internal standards, homogenized, and subjected to protein precipitation. An aliquot of the supernatant was derivatized, then diluted and injected onto liquid chromatography-tandem mass spectrometry, LC-MS/MS system (Agilent 
1290 LC system, Agilent Technologies Inc, Santa Clara, USA with AB Sciex QTrap 5500 system, AB Sciex, Framingham, USA). The mass spectrometer was operated in negative mode using electrospray ionization (ESI). The peak area of the individual analyte product ions was measured against the peak area of the product ions of the corresponding internal standards. Quantification was performed using a weighted linear least squares regression analysis generated from fortified calibration standards prepared immediately prior to each run. LC-MS/MS raw data are collected and processed using AB SCIEX software Analyst 1.6.2.

Global metabolite profiling. Samples were prepared by automated Microlab STAR (Hamilton company, Reno, USA) system [76]. Metabolon inc. used ultraperformance liquid chromatography-tandem mass spectroscopy, UPLC-MS/MS (UPLC from Waters ACQUITY, Milford, USA and Q-Exactive mass spectrometer from Thermo Scientific, Waltham, USA), for the metabolite analysis. After protein precipitation, the resulting extract was aliquoted and two aliquots were analyzed by separate reverse phase (RP)/UPLC-MS/MS methods with positive mode using ESI; one aliquot with RP/UPLC-MS/MS with negative mode using ESI; and one aliquot by hydrophilic interaction chromatography (HILIC)/UPLCMS/MS with negative mode using ESI. Several controls were analyzed in concert with the experimental samples including a pooled matrix sample (and/or a pool of well-characterized human plasma) served as a technical replicate throughout the data set; extracted water samples served as process blanks; and a cocktail of QC standards (carefully selected not to interfere with the spiked endogenous compound into all the samples) to monitor instrument performance and aid in chromatographic alignment. Instrument variability and overall process variability were determined respectively by the standards and spiked endogenous compounds.

Raw data was extracted, peak-identified and QC processed using hardware and software developed by Metabolon [76, 77]. Metabolites were identified by automated comparison of the ion features in the experimental samples to a reference library of chemical standard entries that included retention time, molecular weight $(\mathrm{m} / \mathrm{z})$, preferred adducts, and in-source fragments as well as associated MS spectra and were quality controlled and curated to identified true chemical entities [76, 77]. Peaks were quantified using area-under-the-curve. Data normalization step was performed to correct variation resulting from instrument inter-day tuning differences.

SCFA and metabolite data analysis. Statistical analysis of changes in SCFA concentrations among the treatments were carried out using one-way ANOVA followed by Tukey HSD test after checking for the fulfillment of all pertinent assumptions for ANOVA. On the other hand, Welch's $t$-test which allows for unequal variances was used to analyze changes in metabolite concentrations among the treatments flowing log transformation and imputation of missing values. Changes in SCFAs or metabolite concentrations considered statistically significant when $\mathrm{p} \leq 0.05$.

\section{Correlation analysis of microbiota and metabolites}

Correlation analysis of microbiota and metabolites was performed using M2IA online tool [78]. As per the requirement of the tool, only the metabolites with HMDB IDs (436 and 293 respectively for digesta and 
plasma), and ASV table with taxonomic annotations and corresponding reference sequence file generated from QIIME2 analysis were used. Data was processed by filtering out both the microbiota and metabolic features with missing values found in more than $80 \%$ of samples and the relative standard deviation values less than $30 \%$. Minimum value was selected to impute missing value for both data sets. For data normalization, the relative percentage of features calculated based on the total sum scaling was used. For the pair-wise comparisons of the treatments, Wilcoxon rank-sum test was used and the $p<0.05$ was considered as statistically significant.

Spearman correlation analysis method was selected to analyze correlations between differentially abundant microbiota (genus level) and metabolite concentrations in one dietary group compared to the other. The coefficient values $(R)$ ranged between -1 and 1 and $p<0.05$ was considered statistically significant. The results were visualized on circos plots and heatmaps to identify bacterial genera that were closely related with different classes of metabolites.

Supervised multivariate analysis first integrates two data matrix and then identifies differential variables which significantly contribute to the discrimination between two treatments. We selected the orthogonal partial least squares discriminant analysis (OPLS-DA) method in M2IA to identify the microbiota and metabolites having a significant role in discriminating one dietary group from the other. Variables of importance for group separation were identified and clarified with variable importance plot. Variables with VIP $>1$ and correlation coefficient (corr.coeffs) $p<0.05$ were considered statistically significant.

\section{Declarations}

\section{Ethics approval and consent to participate}

All experiments involving Atlantic salmon were conducted in agreement with the guidelines provided by the Norwegian Animal Research Authority.

\section{Consent for publication}

Not applicable.

\section{Availability of data and materials}

16S rRNA sequencing and RNA-Seq data are publicly available at the NCBI Sequence Read Archive (SRA) with the accession numbers SUB8676898 and SUB8572237 respectively, under the Bioproject PRJNA679207

\section{Competing interests}

The authors reported no potential conflicts of interest.

\section{Funding}


This work was supported by the Norwegian Research Council through a research project (GutBiom project, NFR 281807) and BioMar RD, Trondheim, Norway.

\section{Authors' contributions}

Experiment design: TMK, TF, ÅK and AJT. Providing reagents and materials: TF and TMK. Laboratory work and data analyses: AD, AJT and EC. Writing, original draft: AD. Writing, review and editing: AD, AJT, EC, TMK, TF and ÅK.

\section{Acknowledgements}

The authors would like to thank the employees at LetSea for conducting the feeding trial and preparations for sampling. We are also grateful to Ellen Hage at NBMU (Oslo, Norway) for her skillful organization in sample collection and technical assistance in the laboratory. We would also like to thank Kirsti E. Præsteng at NMBU (Oslo, Norway) for performing 16S rRNA sequencing.

\section{Author details}

${ }^{1}$ Department of Paraclinical Sciences, Faculty of Veterinary Medicine, Norwegian University of Life Sciences (NMBU), Ås, Norway and ${ }^{2}$ Biomar RD, Trondheim, Norway

\section{References}

1. Merrifield DL, Dimitroglou A, Foey A, Davies SJ, Baker RTM, Bøgwald J, et al. The current status and future focus of probiotic and prebiotic applications for salmonids. Aquaculture. 2010;302(1):1-18; doi: https://doi.org/10.1016/j.aquaculture.2010.02.007.

2. Ring $\varnothing$ E, Song SK. Application of dietary supplements (synbiotics and probiotics in combination with plant products and $\beta$-glucans) in aquaculture. Aquac Nutr. 2016;22(1):4-24; doi: 10.1111/anu.12349.

3. Ring $\varnothing \mathrm{E}$, Olsen RE, Gifstad Tø, Dalmo RA, Amlund H, Hemre G-I, et al. Prebiotics in aquaculture: a review. Aquac Nutr. 2010;16(2):117-36; doi: 10.1111/j.1365-2095.2009.00731.x.

4. Song SK, Beck BR, Kim D, Park J, Kim J, Kim HD, et al. Prebiotics as immunostimulants in aquaculture: A review. Fish Shellfish Immunol. 2014;40(1):40-8; doi: https://doi.org/10.1016/j.fsi.2014.06.016.

5. Huynh T-G, Shiu Y-L, Nguyen T-P, Truong Q-P, Chen J-C, Liu C-H. Current applications, selection, and possible mechanisms of actions of synbiotics in improving the growth and health status in aquaculture: A review. Fish Shellfish Immunol. 2017;64:367-82; doi: https://doi.org/10.1016/j.fsi.2017.03.035.

6. Rahimnejad S, Guardiola FA, Leclercq E, Ángeles Esteban M, Castex M, Sotoudeh E, et al. Effects of dietary supplementation with Pediococcus acidilactici MA18/5M, galactooligosaccharide and their synbiotic on growth, innate immunity and disease resistance of rockfish (Sebastes schlegeli). Aquaculture. 2018;482:36-44; doi: https://doi.org/10.1016/j.aquaculture.2017.09.020. 
7. Abid A, Davies SJ, Waines P, Emery M, Castex M, Gioacchini G, et al. Dietary synbiotic application modulates Atlantic salmon (Salmo salar) intestinal microbial communities and intestinal immunity. Fish Shellfish Immunol. 2013;35(6):1948-56; doi: https://doi.org/10.1016/j.fsi.2013.09.039.

8. Merrifield DL, Bradley G, Harper GM, Baker RTM, Munn CB, Davies SJ. Assessment of the effects of vegetative and lyophilized Pediococcus acidilactici on growth, feed utilization, intestinal colonization and health parameters of rainbow trout (Oncorhynchus mykiss Walbaum). Aquac Nutr. 2011;17(1):73-9; doi: 10.1111/j.1365-2095.2009.00712.x.

9. Ferguson RMW, Merrifield DL, Harper GM, Rawling MD, Mustafa S, Picchietti S, et al. The effect of Pediococcus acidilactici on the gut microbiota and immune status of on-growing red tilapia (Oreochromis niloticus). J Appl Microbiol. 2010;109(3):851-62; doi: 10.1111/j.13652672.2010.04713.x.

10. Jaramillo-Torres A, Rawling MD, Rodiles A, Mikalsen HE, Johansen L-H, Tinsley J, et al. Influence of dietary supplementation of probiotic Pediococcus acidilactici MA18/5M during the transition from freshwater to seawater on intestinal health and microbiota of Atlantic slmon (Salmo salarL.). Front Microbiol. 2019;10(2243); doi: 10.3389/fmicb.2019.02243.

11. Kiron V, Kulkarni A, Dahle D, Vasanth G, Lokesh J, Elvebo O. Recognition of purified beta 1,3/1,6 glucan and molecular signalling in the intestine of Atlantic salmon. Dev Comp Immunol. 2016;56:5766; doi: 10.1016/j.dci.2015.11.007.

12. Wang J, Kortner TM, Chikwati EM, Li Y, Jaramillo-Torres A, Jakobsen JV, et al. Gut immune functions and health in Atlantic salmon (Salmo salar) from late freshwater stage until one year in seawater and effects of functional ingredients: A case study from a commercial sized research site in the Arctic region. Fish Shellfish Immunol. 2020;106:1106-19; doi: https://doi.org/10.1016/j.fsi.2020.09.019.

13. Gibson GR, Roberfroid MB. Dietary modulation of the human colonic microbiota: introducing the concept of prebiotics. J Nutr. 1995;125(6):1401-12; doi: 10.1093/jn/125.6.1401.

14. Markowiak P, Śliżewska K. Effects of probiotics, prebiotics, and synbiotics on human health. Nutrients. 2017;9(9):1021.

15. Aftabgard M, Salarzadeh A, Mohseni M. The effects of a synbiotic mixture of galactooligosaccharides and Bacillus strains in Caspian salmon, Salmo trutta caspius Fingerlings. Probiotics Antimicrob Proteins. 2019;11(4):1300-8; doi: 10.1007/s12602-018-9498-4.

16. Hoseinifar SH, Yousefi S, Van Doan H, Ashouri G, Gioacchini G, Maradonna F, et al. Oxidative stress and antioxidant defense in fish: the implications of probiotic, prebiotic, and synbiotics. Rev Fish Sci Aquacult. 2020:1-20; doi: 10.1080/23308249.2020.1795616.

17. Hoseinifar SH, Mirvaghefi A, Amoozegar MA, Sharifian M, Esteban MÁ. Modulation of innate immune response, mucosal parameters and disease resistance in rainbow trout (Oncorhynchus mykiss) upon synbiotic feeding. Fish Shellfish Immunol. 2015;45(1):27-32; doi: https://doi.org/10.1016/j.fsi.2015.03.029. 
18. Hoseinifar SH, Hoseini SM, Bagheri D. Effects of galactooligosaccharide and Pediococcus acidilactici on antioxidant defence and disease resistance of Rainbow trout, Oncorhynchus Mykiss. Ann Anim Sci. 2017;17(1):217; doi: https://doi.org/10.1515/aoas-2016-0024.

19. Rasmussen JA, Villumsen KR, Ernst M, Hansen M, Forberg T, Gopalakrishnan S, et al. A multi-omics approach unravels metagenomic and metabolic alterations of a probiotic and synbiotic additive in rainbow trout (Oncorhynchus mykiss). Microbiome. 2022;10(1):21; doi: 10.1186/s40168-021-012218.

20. Modanloo M, Soltanian S, Akhlaghi M, Hoseinifar SH. The effects of single or combined administration of galactooligosaccharide and Pediococcus acidilactici on cutaneous mucus immune parameters, humoral immune responses and immune related genes expression in common carp (Cyprinus carpio) fingerlings. Fish Shellfish Immunol. 2017;70:391-7; doi: 10.1016/j.fsi.2017.09.032.

21. Soltani M, Badzohreh G, Mirzargar S, Farhangi M, Shekarabi PH, Lymbery A. Growth behavior and fatty acid production of probiotics, Pediococcus acidilactici and Lactococcus lactis, at different concentrations of fructooligosaccharide: studies validating clinical efficacy of selected synbiotics on growth performance of Caspian roach (Rutilus frisii kutum) Fry. Probiotics Antimicrob Proteins. 2019;11(3):765-73; doi: 10.1007/s12602-018-9462-3.

22. FAO: The state of world fisheries and aquaculture 2020. In. Rome, Italy; 2020.

23. Thorstad EB, Whoriskey F, Uglem I, Moore A, Rikardsen AH, Finstad B. A critical life stage of the Atlantic salmon Salmo salar. behaviour and survival during the smolt and initial post-smolt migration. J Fish Biol. 2012;81(2):500-42; doi: 10.1111/j.1095-8649.2012.03370.x.

24. Wang J, Jaramillo-Torres A, Li Y, Kortner TM, Gajardo K, Brevik ØJ, et al. Microbiota in intestinal digesta of Atlantic salmon (Salmo salar), observed from late freshwater stage until one year in seawater, and effects of functional ingredients: a case study from a commercial sized research site in the Arctic region. Anim Microbiome. 2021;3(1):14; doi: 10.1186/s42523-021-00075-7.

25. Li Y, Bruni L, Jaramillo-Torres A, Gajardo K, Kortner TM, Krogdahl Å. Differential response of digestaand mucosa-associated intestinal microbiota to dietary insect meal during the seawater phase of Atlantic salmon. Anim Microbiome. 2021;3(1):8; doi: 10.1186/s42523-020-00071-3.

26. Gajardo K, Jaramillo-Torres A, Kortner TM, Merrifield DL, Tinsley J, Bakke AM, et al. Alternative protein sources in the diet modulate microbiota and functionality in the distal intestine of Atlantic salmon (Salmo salar). Appl Environ Microbiol. 2017;83(5); doi: 10.1128/aem.02615-16.

27. Gajardo K, Rodiles A, Kortner TM, Krogdahl Å, Bakke AM, Merrifield DL, et al. A high-resolution map of the gut microbiota in Atlantic salmon (Salmo salar): A basis for comparative gut microbial research. Sci Rep. 2016;6:30893-; doi: 10.1038/srep30893.

28. Vernocchi P, Del Chierico F, Putignani L. Gut microbiota profiling: metabolomics based approach to unravel compounds affecting human health. Front Microbiol. 2016;7(1144); doi: 10.3389/fmicb.2016.01144.

29. Holmes E, Li JV, Athanasiou T, Ashrafian H, Nicholson JK. Understanding the role of gut microbiomehost metabolic signal disruption in health and disease. Trends Microbiol. 2011;19(7):349-59; doi: 
10.1016/j.tim.2011.05.006.

30. Hoseinifar SH, Mirvaghefi A, Amoozegar MA, Merrifield DL, Ring $\varnothing$ E. In vitro selection of a synbiotic and in vivo evaluation on intestinal microbiota, performance and physiological response of rainbow trout (Oncorhynchus mykiss) fingerlings. Aquac Nutr. 2017;23(1):111-8; doi: https://doi.org/10.1111/anu.12373.

31. Papagianni M, Anastasiadou S. Pediocins: The bacteriocins of Pediococci. Sources, production, properties and applications. Microb Cell Fact. 2009;8:3; doi: 10.1186/1475-2859-8-3.

32. Porto MC, Kuniyoshi TM, Azevedo PO, Vitolo M, Oliveira RP. Pediococcus spp.: An important genus of lactic acid bacteria and pediocin producers. Biotechnol Adv. 2017;35(3):361-74; doi: 10.1016/j.biotechadv.2017.03.004.

33. Bremer J. Carnitine--metabolism and functions. Physiol Rev. 1983;63(4):1420-80; doi: 10.1152/physrev.1983.63.4.1420.

34. Reuter SE, Evans AM. Carnitine and Acylcarnitines. Clin Pharmacokinet. 2012;51(9):553-72; doi: 10.1007/BF03261931.

35. Ghonimy A, Zhang DM, Farouk MH, Wang Q. The impact of carnitine on dietary fiber and gut bacteria metabolism and their mutual interaction in monogastrics. Int J Mol Sci. 2018;19(4):1008; doi: 10.3390/ijms19041008.

36. Slotte JP. Biological functions of sphingomyelins. Prog Lipid Res. 2013;52(4):424-37; doi: https://doi.org/10.1016/j.plipres.2013.05.001.

37. Maceyka M, Spiegel S. Sphingolipid metabolites in inflammatory disease. Nature. 2014;510(7503):58-67; doi: 10.1038/nature13475.

38. Rolando $\mathrm{M}$, Buchrieser $\mathrm{C}$. A Comprehensive review on the manipulation of the sphingolipid pathway by pathogenic bacteria. Front Cell Dev Bio. 2019;7(168); doi: 10.3389/fcell.2019.00168.

39. den Besten G, van Eunen K, Groen AK, Venema K, Reijngoud D-J, Bakker BM. The role of short-chain fatty acids in the interplay between diet, gut microbiota, and host energy metabolism. J Lipid Res. 2013;54(9):2325-40; doi: 10.1194/jlr.R036012.

40. McLoughlin RF, Berthon BS, Jensen ME, Baines KJ, Wood LG. Short-chain fatty acids, prebiotics, synbiotics, and systemic inflammation: a systematic review and meta-analysis. Am J Clin Nutr. 2017;106(3):930-45; doi: 10.3945/ajcn.117.156265.

41. Allameh SK, Ring $\varnothing$ E, Yusoff FM, Daud HM, Ideris A. Dietary supplement of Enterococcus faecalis on digestive enzyme activities, short-chain fatty acid production, immune system response and disease resistance of Javanese carp (Puntius gonionotus, Bleeker 1850). Aquac Nutr. 2017;23(2):331-8; doi: 10.1111/anu.12397.

42. Asaduzzaman M, lehata S, Akter S, Kader MA, Ghosh SK, Khan MNA, et al. Effects of host gut-derived probiotic bacteria on gut morphology, microbiota composition and volatile short chain fatty acids production of Malaysian Mahseer Tor tambroides. Aquac Rep. 2018;9:53-61; doi: https://doi.org/10.1016/j.aqrep.2017.12.003. 
43. Macfarlane S, Macfarlane GT. Regulation of short-chain fatty acid production. Proc Nutr Soc. 2003;62(1):67-72; doi: 10.1079/PNS2002207.

44. Zou J, Secombes CJ. The function of fish cytokines. Biology. 2016;5(2):23; doi: 10.3390/biology5020023.

45. Bird S, Tafalla C. Teleost chemokines and their receptors. Biology. 2015;4(4):756-84; doi: $10.3390 /$ biology 4040756.

46. Shan S, Liu D, Liu R, Zhu Y, Li T, Zhang F, et al. Non-mammalian Toll-like receptor 18 (TIr18) recognizes bacterial pathogens in common carp (Cyprinus carpio L.): Indications for a role of participation in the NF-kB signaling pathway. Fish Shellfish Immunol. 2018;72:187-98; doi: https://doi.org/10.1016/j.fsi.2017.09.081.

47. van der Vliet A, Danyal K, Heppner DE. Dual oxidase: a novel therapeutic target in allergic disease. $\mathrm{Br}$ J Pharmacol. 2018;175(9):1401-18; doi: 10.1111/bph.14158.

48. Bedard K, Krause K-H. The NOX family of ROS-generating NADPH oxidases: physiology and pathophysiology. Physiol Rev. 2007;87(1):245-313; doi: 10.1152/physrev.00044.2005.

49. Semova I, Carten JD, Stombaugh J, Mackey LC, Knight R, Farber SA, et al. Microbiota regulate intestinal absorption and metabolism of fatty acids in the zebrafish. Cell Host Microbe. 2012;12(3):277-88; doi: 10.1016/j.chom.2012.08.003.

50. Falcinelli S, Picchietti S, Rodiles A, Cossignani L, Merrifield DL, Taddei AR, et al. Lactobacillus rhamnosus lowers zebrafish lipid content by changing gut microbiota and host transcription of genes involved in lipid metabolism. Sci Rep. 2015;5:9336; doi: 10.1038/srep09336.

51. Lin R, Liu W, Piao M, Zhu H. A review of the relationship between the gut microbiota and amino acid metabolism. Amino Acids. 2017;49(12):2083-90; doi: 10.1007/s00726-017-2493-3.

52. Rowland I, Gibson G, Heinken A, Scott K, Swann J, Thiele I, et al. Gut microbiota functions: metabolism of nutrients and other food components. Eur J Nutr. 2018;57(1):1-24; doi: 10.1007/s00394-017-1445-8.

53. FEEDAP. Assessment of the application for renewal of authorisation of Bactocell ${ }^{\circledR}$ (Pediococcus acidilactici CNCM I-4622) as a feed additive for all fish and shrimps and its extension of use for all crustaceans. EFSA Journal. 2019;17(4):e05691; doi: 10.2903/j.efsa.2019.5691.

54. Thorarensen H, Farrell AP. The biological requirements for post-smolt Atlantic salmon in closedcontainment systems. Aquaculture. 2011;312(1):1-14; doi: https://doi.org/10.1016/j.aquaculture.2010.11.043.

55. Knudsen BE, Bergmark L, Munk P, Lukjancenko O, Priemé A, Aarestrup FM, et al. Impact of sample type and DNA isolation procedure on genomic inference of microbiome composition. mSystems. 2016;1(5):e00095-16; doi: 10.1128/mSystems.00095-16.

56. Ramseier CA, Kinney JS, Herr AE, Braun T, Sugai JV, Shelburne CA, et al. Identification of pathogen and host-response markers correlated with periodontal disease. J Periodontol. 2009;80(3):436-46; doi: 10.1902/jop.2009.080480. 
57. Vandeputte D, Kathagen G, D'hoe K, Vieira-Silva S, Valles-Colomer M, Sabino J, et al. Quantitative microbiome profiling links gut community variation to microbial load. Nature. 2017;551(7681):50711; doi: 10.1038/nature24460.

58. Rasmussen R. Quantification on the LightCycler. In: S. Meuer S, C. Wittwer C, Nakagawara KI, editors. Rapid Cycle Real-Time PCR. Berlin, Heidelberg: Springer; 2001.

59. Hellemans J, Mortier G, De Paepe A, Speleman F, Vandesompele J. qBase relative quantification framework and software for management and automated analysis of real-time quantitative PCR data. Genome Biol. 2007;8(2):R19; doi: 10.1186/gb-2007-8-2-r19.

60. Bolyen E, Rideout JR, Dillon MR, Bokulich NA, Abnet CC, Al-Ghalith GA, et al. Reproducible, interactive, scalable and extensible microbiome data science using QIIME 2. Nat Biotechnol. 2019;37(8):852-7; doi: 10.1038/s41587-019-0209-9.

61. Caporaso JG, Kuczynski J, Stombaugh J, Bittinger K, Bushman FD, Costello EK, et al. QIIME allows analysis of high-throughput community sequencing data. Nat Methods. 2010;7(5):335-6; doi: 10.1038/nmeth.f.303.

62. Callahan BJ, McMurdie PJ, Rosen MJ, Han AW, Johnson AJ, Holmes SP. DADA2: High-resolution sample inference from Illumina amplicon data. Nat Methods. 2016;13(7):581-3; doi: 10.1038/nmeth.3869.

63. Bokulich NA, Kaehler BD, Rideout JR, Dillon M, Bolyen E, Knight R, et al. Optimizing taxonomic classification of marker-gene amplicon sequences with QIIME 2's q2-feature-classifier plugin. Microbiome. 2018;6(1):90; doi: 10.1186/s40168-018-0470-z.

64. Quast C, Pruesse E, Yilmaz P, Gerken J, Schweer T, Yarza P, et al. The SILVA ribosomal RNA gene database project: improved data processing and web-based tools. Nucleic Acids Res. 2013;41(Database issue):D590-6; doi: 10.1093/nar/gks1219.

65. Davis NM, Proctor DM, Holmes SP, Relman DA, Callahan BJ. Simple statistical identification and removal of contaminant sequences in marker-gene and metagenomics data. Microbiome. 2018;6(1):226; doi: 10.1186/s40168-018-0605-2.

66. Dhariwal A, Chong J, Habib S, King IL, Agellon LB, Xia J. MicrobiomeAnalyst: a web-based tool for comprehensive statistical, visual and meta-analysis of microbiome data. Nucleic Acids Res. 2017;45(W1):W180-W8; doi: 10.1093/nar/gkx295.

67. Chong J, Liu P, Zhou G, Xia J. Using MicrobiomeAnalyst for comprehensive statistical, functional, and meta-analysis of microbiome data. Nat Protoc. 2020;15(3):799-821; doi: 10.1038/s41596-0190264-1.

68. Martin M. Cutadapt removes adapter sequences from high-throughput sequencing reads. EMBnet J. 2011;17(1); doi: 10.14806/ej.17.1.200.

69. Kim D, Paggi JM, Park C, Bennett C, Salzberg SL. Graph-based genome alignment and genotyping with HISAT2 and HISAT-genotype. Nat Biotechnol. 2019;37(8):907-15; doi: 10.1038/s41587-0190201-4. 
70. Tekle KM, Gundersen S, Klepper K, Bongo LA. Norwegian e-Infrastructure for Life Sciences (NeLS). F1000Research. 2018;7(ELIXIR):968; doi: https://doi.org/10.12688/f1000research.15119.1.

71. Anders S, Pyl PT, Huber W. HTSeq-a Python framework to work with high-throughput sequencing data. Bioinformatics. 2014;31(2):166-9; doi: 10.1093/bioinformatics/btu638.

72. Anders S, Huber W. Differential expression analysis for sequence count data. Genome Biol. 2010;11(10):1-12; doi: 10.1186/gb-2010-11-10-r106.

73. Reimand J, Kull M, Peterson H, Hansen J, Vilo J. g:Profiler-a web-based toolset for functional profiling of gene lists from large-scale experiments. Nucleic Acids Res. 2007;35(Web Server issue):W193-W200; doi: 10.1093/nar/gkm226.

74. Raudvere U, Kolberg L, Kuzmin I, Arak T, Adler P, Peterson H, et al. g:Profiler: a web server for functional enrichment analysis and conversions of gene lists (2019 update). Nucleic Acids Res. 2019;47(W1):W191-W8; doi: 10.1093/nar/gkz369.

75. Supek F, Bošnjak M, Škunca N, Šmuc T. REVIGO summarizes and visualizes long lists of gene ontology terms. PLoS One. 2011;6(7):e21800; doi: 10.1371/journal.pone.0021800.

76. Evans AM, DeHaven CD, Barrett T, Mitchell M, Milgram E. Integrated, Nontargeted Ultrahigh Performance Liquid Chromatography/Electrospray Ionization Tandem Mass Spectrometry Platform for the Identification and Relative Quantification of the Small-Molecule Complement of Biological Systems. Anal Chem. 2009;81(16):6656-67; doi: 10.1021/ac901536h.

77. Evans AM, Br B, Liu Q, Mitchell MW, Rj R, Dai H, et al. High Resolution Mass Spectrometry Improves Data Quantity and Quality as Compared to Unit Mass Resolution Mass Spectrometry in HighThroughput Profiling Metabolomics. Metabolomics. 2014;4:1-3.

78. Ni Y, Yu G, Chen H, Deng Y, Wells PM, Steves CJ, et al. M2IA: a web server for microbiome and metabolome integrative analysis. Bioinformatics. 2020;36(11):3493-8; doi:

10.1093/bioinformatics/btaa188.

\section{Figures}




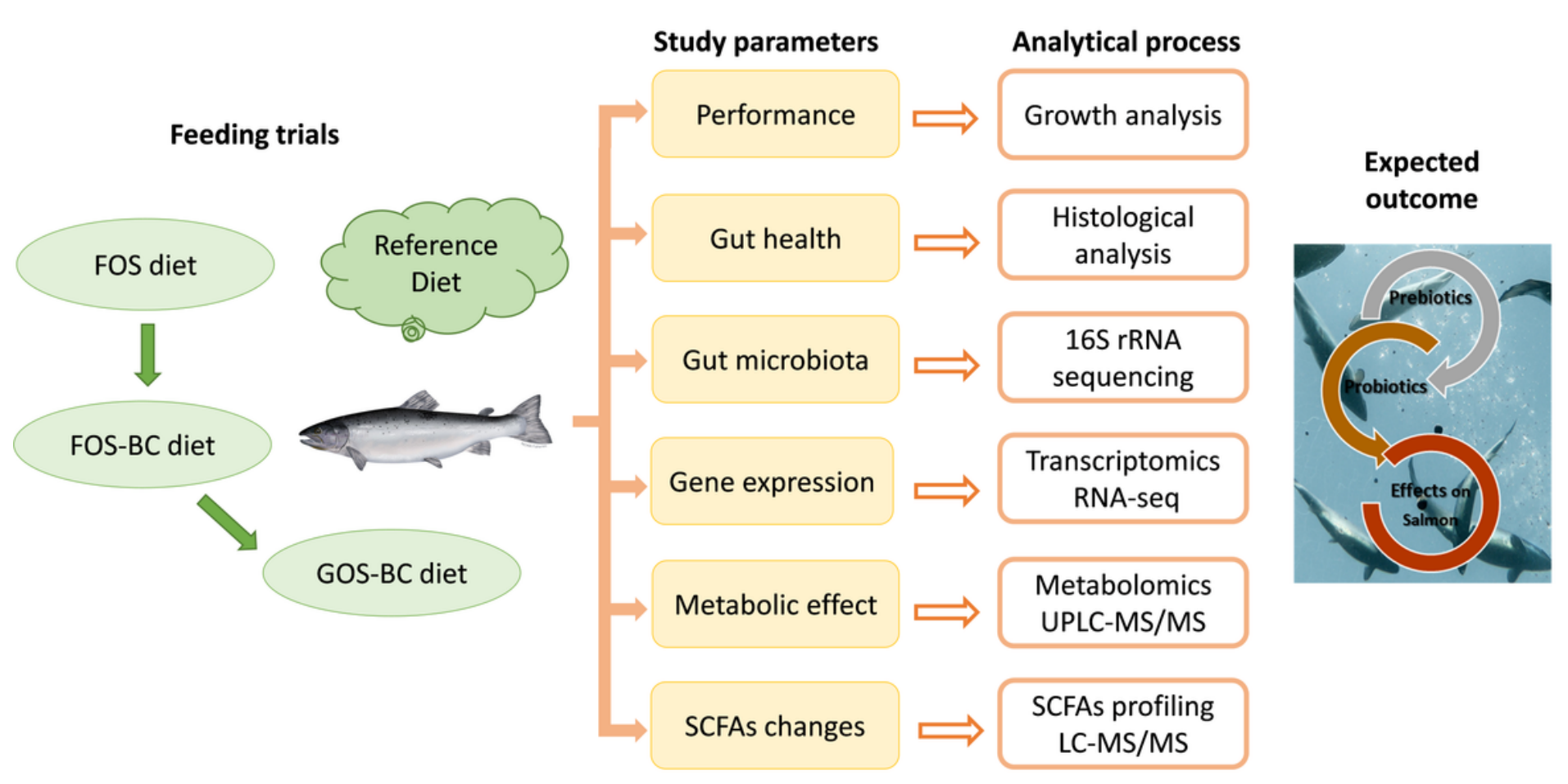

\section{Figure 1}

Schematic representation of experimental design used to evaluate the effects of supplementation of $P$. acidilactici in diets for Atlantic salmon performance and gut health after transfer from freshwater to seawater. Fish were fed FOS alone (FOS diet) and FOS and GOS in combination with P. acidilactici (FOS$\mathrm{BC}$ and GOS-BC diets respectively) and a commercial diet as a control/reference for 10 weeks. Six different parameters were analyzed using traditional and state-of-art-multi-omics techniques as detailed in the materials and methods section to investigate the effects of supplemented diets on post-smolt Atlantic salmon. Photograph. Geir Mogen, BioMar. 


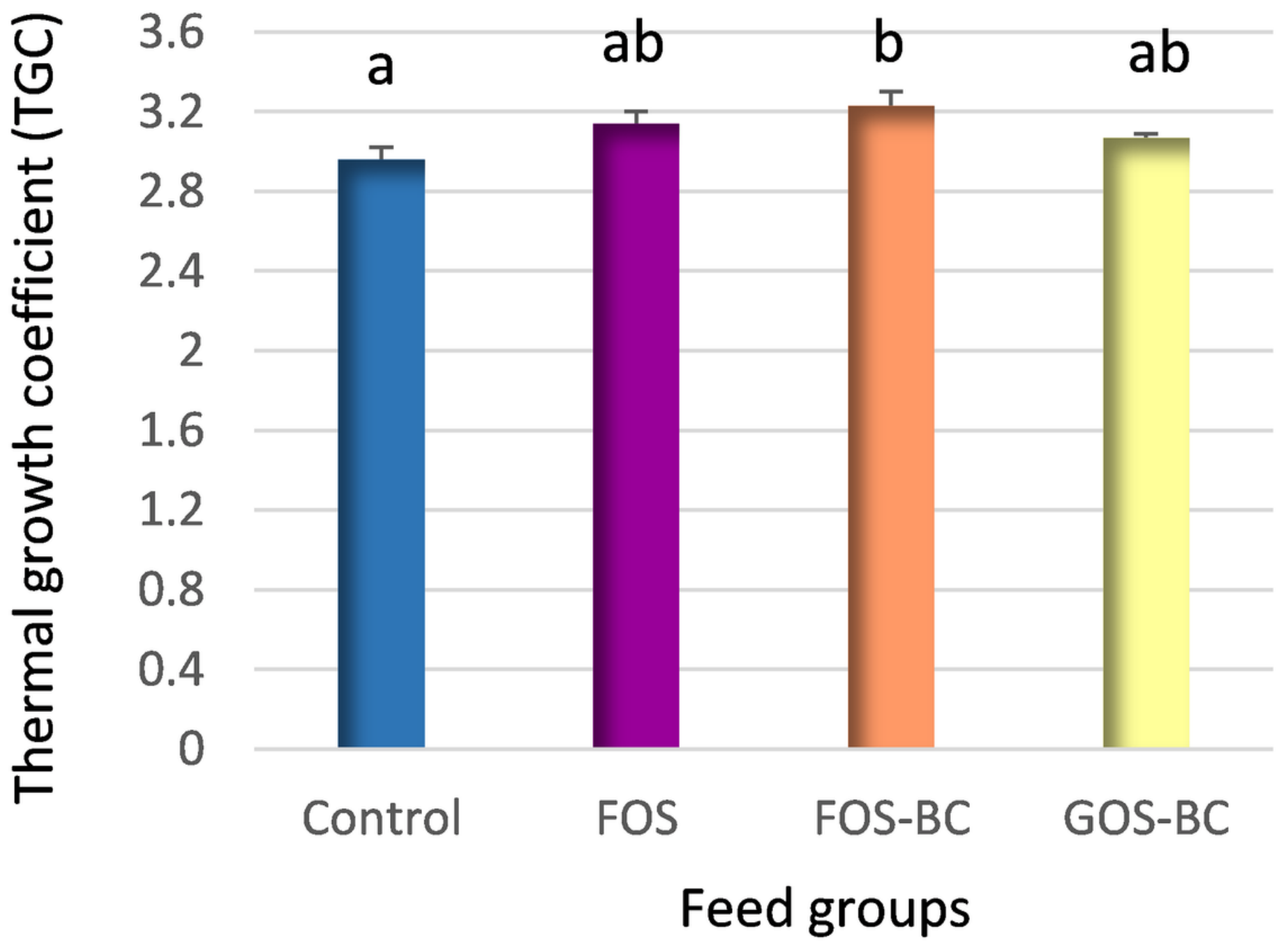

\section{$\square$ Control $\square$ FOS $\square$ FOS-BC $\square$ GOS-BC}

Figure 2

The thermal growth coefficient (TGC) of Atlantic salmon fed a commercial diet as a control/reference and three experimental diets: FOS alone (FOS diet) and FOS and GOS in combination with $P$. acidilactici (FOS$B C$ and GOS-BC diets respectively) for 10 weeks. Values are means \pm SEM $(n=$ average of 210 fish per group). Different letters indicate statistically significant difference $(p \leq 0.05)$ among treatments. 
(a) DI mucosal cell infiltration

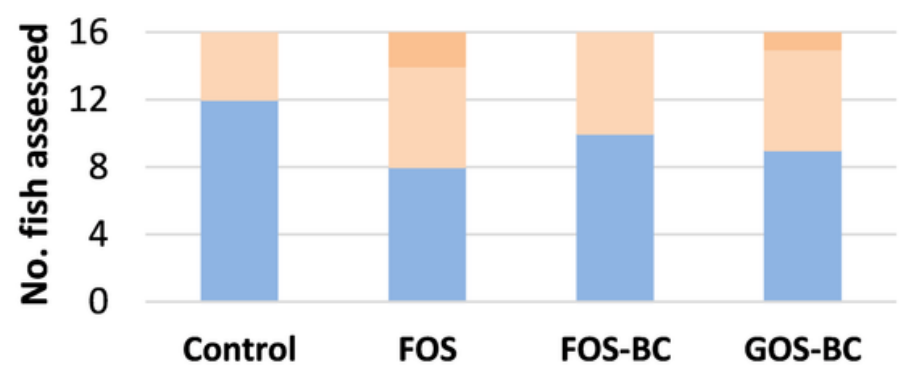

(c) PC mucosal cell inflitration

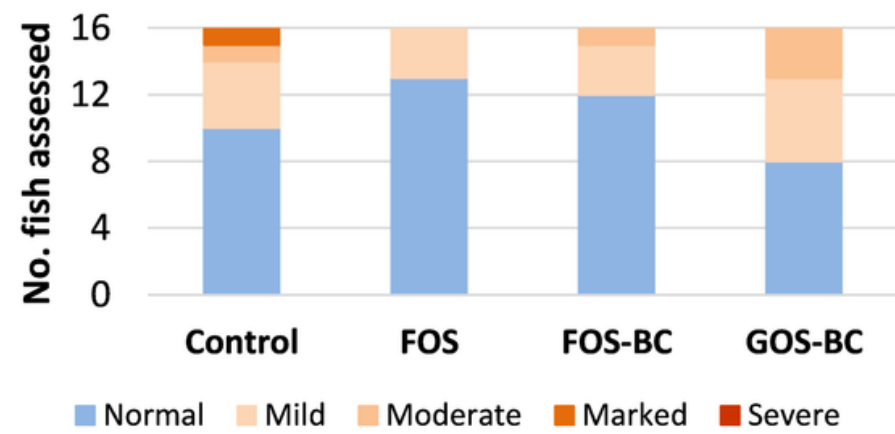

(b) Loss in DI enterocyte vacuolization

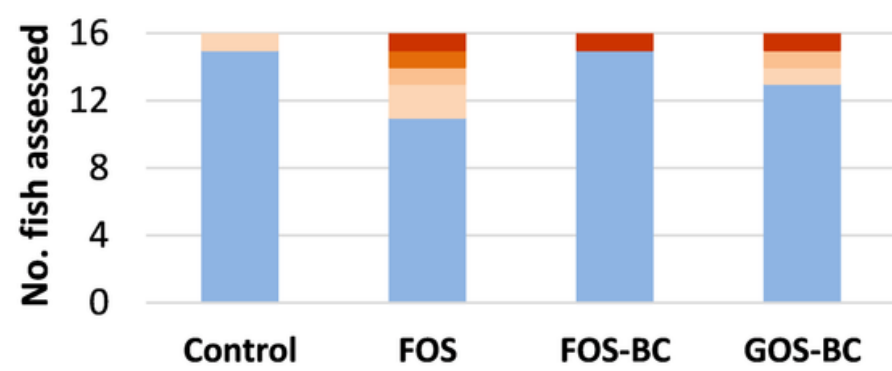

(d) PC enterocyte steatosis

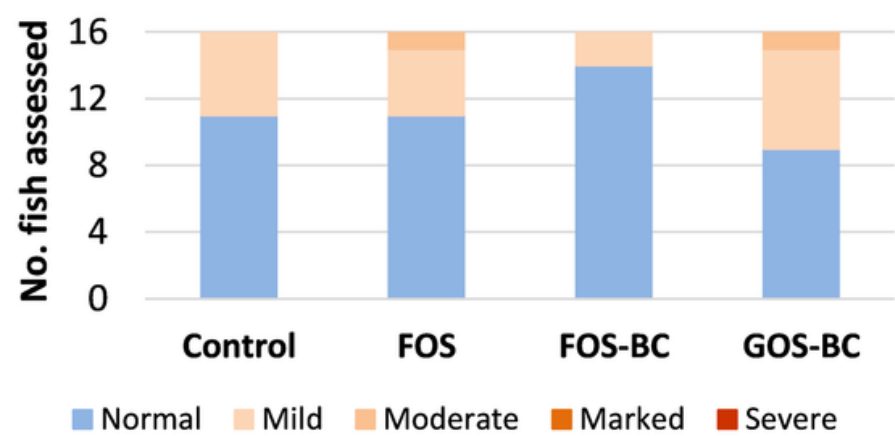

Figure 3

Number of fish scored as normal, mild moderate, marked, or severe for selected histomorphological of (a) distal intestine inflammatory cell infiltration $(p=0.638)$, (b) loss of distal intestine enterocyte vacuoles ( $p$ $=0.097)$, (c) inflammatory cell infiltration of the pyloric caeca mucosa $(p=0.529)$, and (d) lipid accumulation (steatosis) in pyloric caeca enterocytes $(p=0.437)$. $p$-values represent outcomes of an ordinal logistic regression for differences in histology score outcomes between the treatment and the reference group, Control.

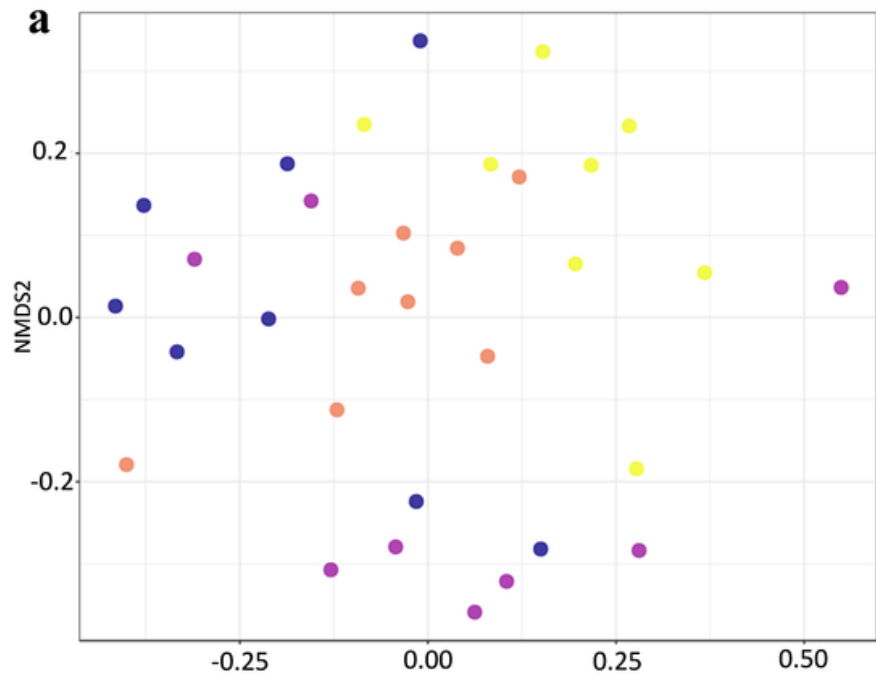

[PERMANOVA] F-value: 1.9; R-squared: 0.17; p-value $<0.04$

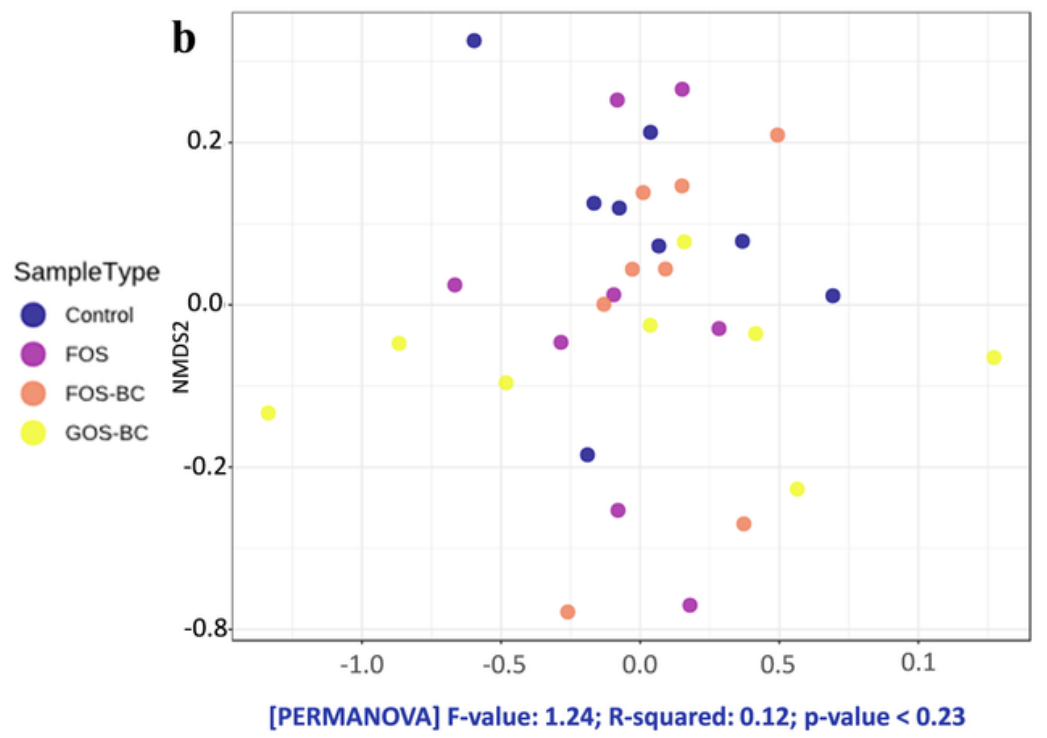




\section{Figure 4}

NMDs plots based on Bray-Curtis dissimilarity matrix showing bacterial community structures (beta diversity) in the distal intestine (a) digesta and (b) mucosa of the Atlantic salmon fed with a control/reference diet and three experimental diets; FOS, FOS-BC and GOS-BC. The whole bacterial community of each sample is represented by a dot on the figure. Samples with similar bacterial compositions are closer to each other.
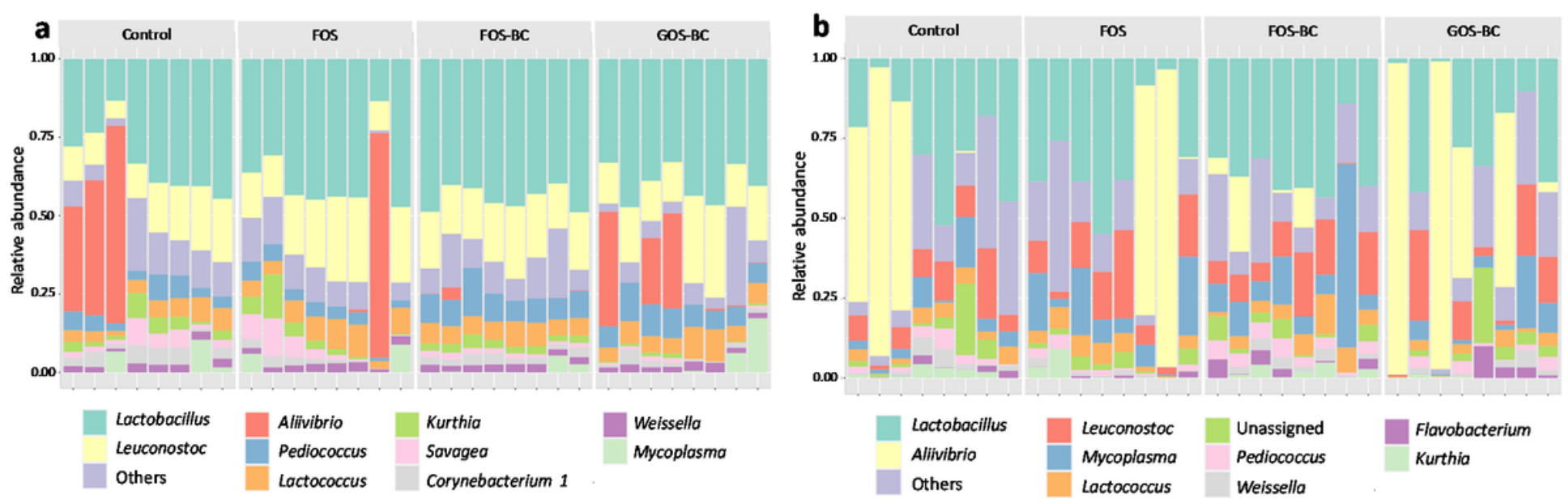

\section{Figure 5}

Ten most abundant genera of digesta (a) and mucosa (b) from distal intestine of the Atlantic salmon fed with a control/reference diet and three experimental diets; FOS, FOS-BC and GOS-BC diets. 

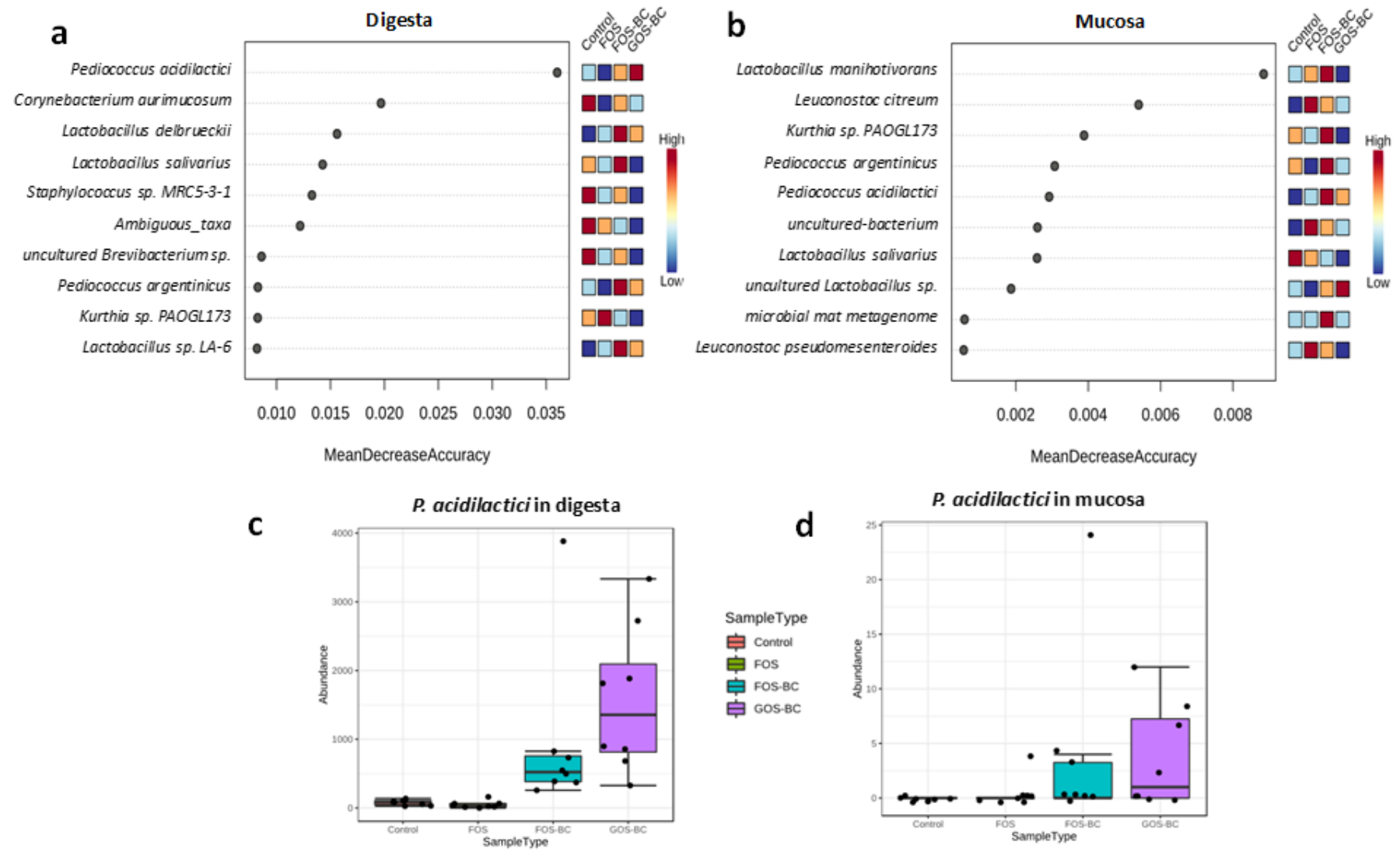

\section{Figure 6}

Random Forest importance plot indicating top ten microbial species in digesta (a) and mucosa (b) valuable for discriminating four treatments. The importance of the microbiota is ordered from top to bottom and an estimate of their importance is indicated by the corresponding mean decrease accuracy. Box plots showing filtered absolute counts of $P$. acidilactici in digesta (c) and mucosa (d) which is important for separating fish in FOS-BC and GOS-BC from those in the control and FOS treatments. 

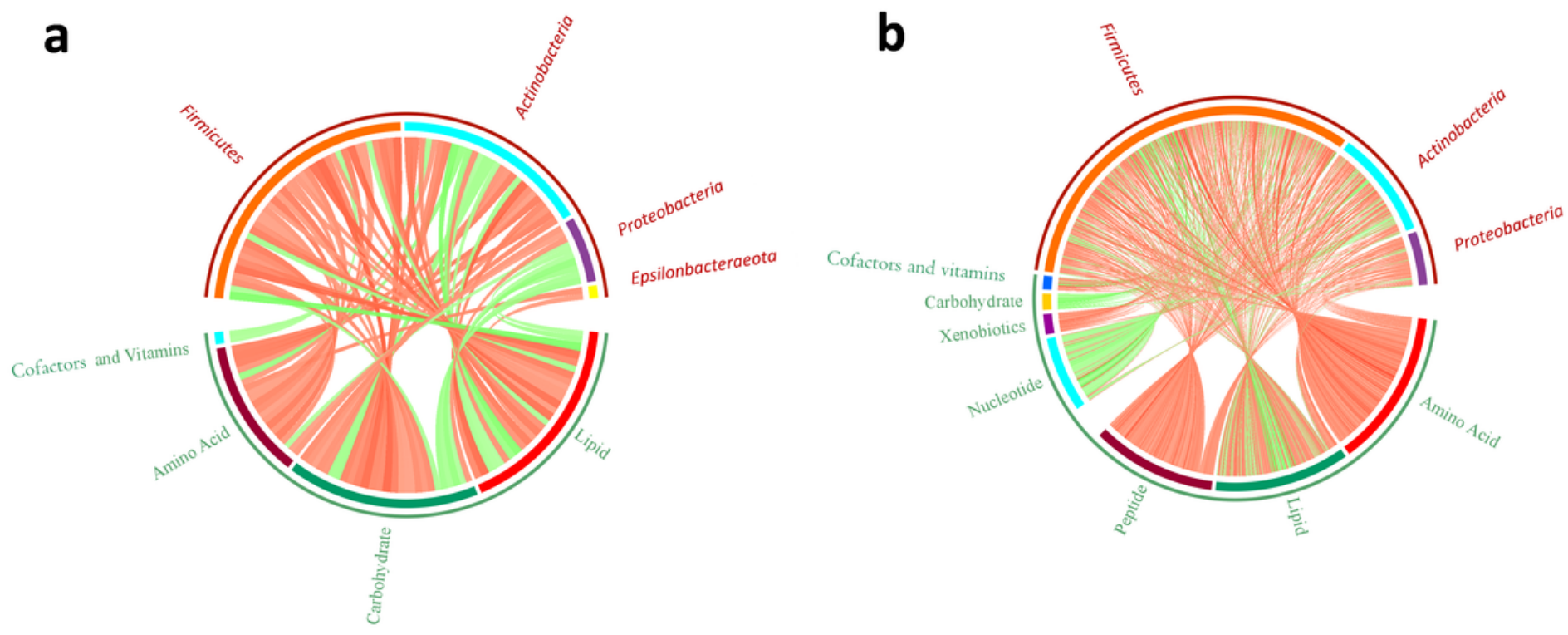

Figure 7

Circos plots showing correlations between differential microbiota and metabolite classes in FOS-BC group compared to FOS group (a) and GOS-BC group compared to FOS-BC group (b) based on Spearman Correlation Analysis. Spearman's correlation, $R$, ranges between -1 to 1. $p<0.05$ indicates a statistically significant correlation. Red or green lines specify positive or negative correlations.
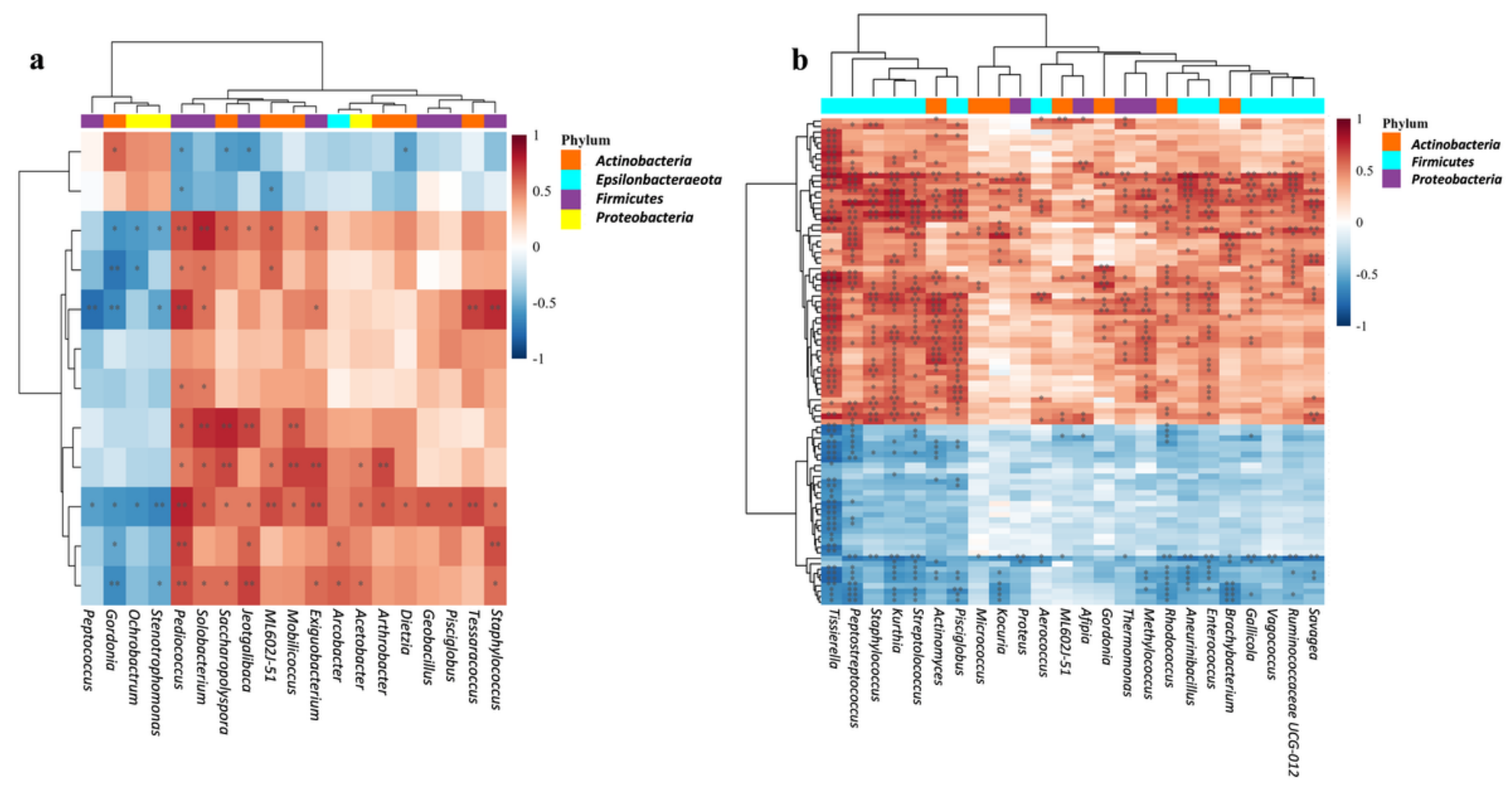

Figure 8 
Heat maps showing correlations between differential microbiota and metabolites in FOS-BC group compared to FOS group (a) and GOS-BC group compared to FOS-BC group (b) based on Spearman Correlation Analysis. Spearman's correlation, $R$, ranges between -1 to 1. $p<0.05$ indicates a statistically significant correlation. Red color and blue color indicate positive and negative correlations respectively. The darker color indicates the larger statistical significance. Symbol * and ** indicate p-value for correlation coefficients smaller than 0.05 or 0.01 , respectively. Correlations between differential microbiota and metabolites among the treatments including $\mathrm{R}$ and $\mathrm{p}$-values presented in Additional File 4.

a
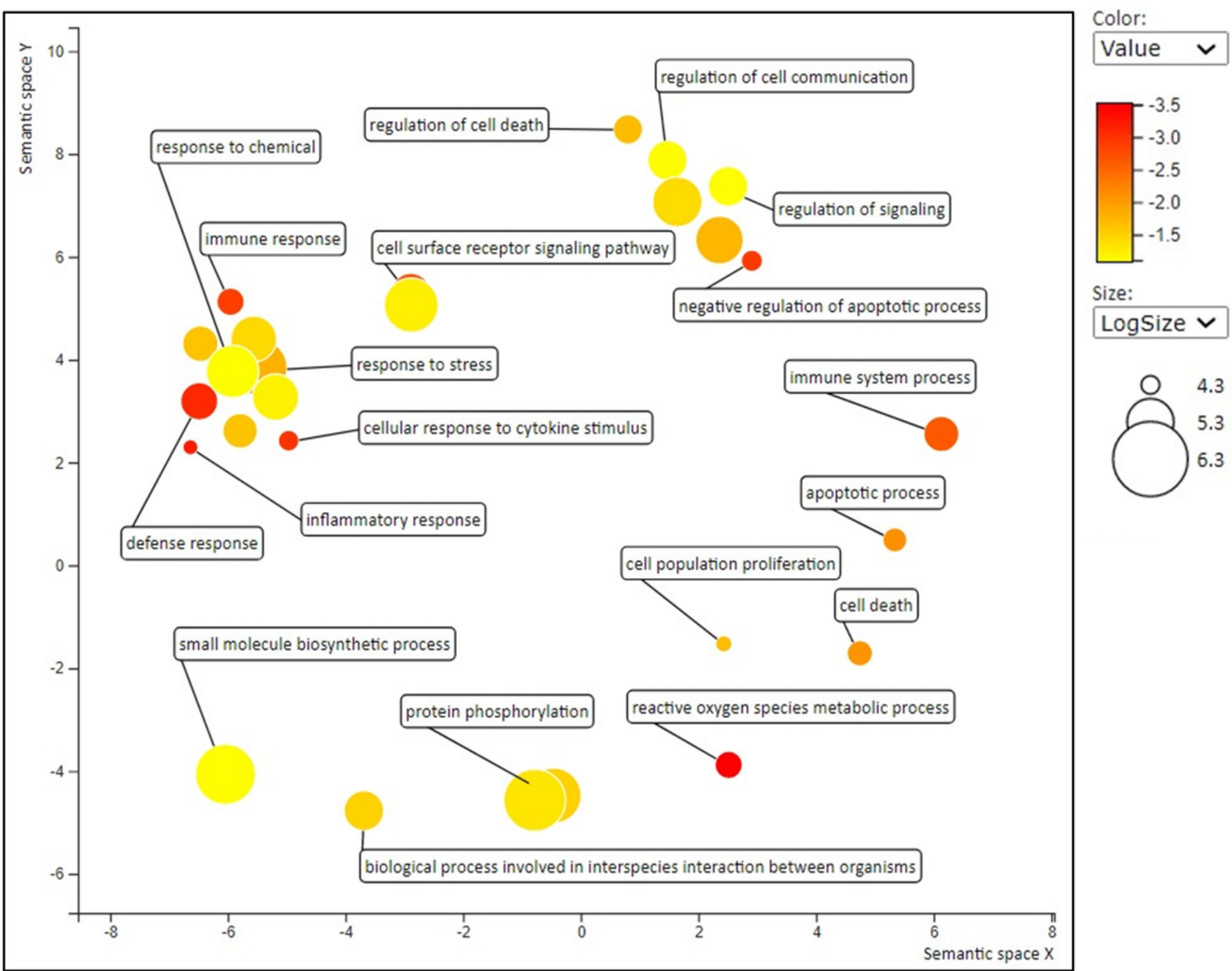

\section{Figure 9}

Non-redundant enriched gene ontology (GO) biological processes determined for the upregulated genes in Atlantic salmon fed the GOS-BC treatment compared to fish fed the FOS-BC treatment. Data are summarized as scatter plots using REVIGO tool. GO terms are marked with circles and plotted according 
to semantic similarities to other $\mathrm{GO}$ terms. The color of the circles ranging from yellow to red indicates the order of increase in log10 p-value. Circle sizes are proportional to the respective frequencies of the GO terms (circles of more general terms are larger). Not all the terms are indicated in the figure due to the space limitations and the complete list of non-redundant enriched GO terms can be found in Additional File 2: Table S6.

\section{Supplementary Files}

This is a list of supplementary files associated with this preprint. Click to download.

- DhanasirietalAdditionalFile1.docx

- DhanasirietalAdditionalFile2.docx

- DhanasirietalAdditionalFile3.xlsx

- DhanasirietalAddtionalFile4.xIsx

- DhanasirietalAdditionalFile5.xlsx 